\title{
Layering and turbulence surrounding an anticyclonic oceanic vortex: in situ observations and quasi-geostrophic numerical simulations
}

\author{
Bach Lien Hua $^{1}{ }^{\dagger}$, Claire Ménesguen ${ }^{1}{ }^{\dagger}$, Sylvie Le Gentil ${ }^{1}$, Richard Schopp ${ }^{1}$, \\ Bruno Marsset ${ }^{2}$ and Hidenori Aiki ${ }^{3}$ \\ ${ }^{1}$ Laboratoire de Physique des Oceans, IFREMER-CNRS, BP 70, 29280 Plouzané, France \\ ${ }^{2}$ Laboratoire de Géophysique et Géodynamique, IFREMER, BP 70, 29280 Plouzané, France \\ ${ }^{3}$ JAMSTEC, Yokohama 236-0001, Japan
}

(Received 29 May 2012; revised 8 July 2013; accepted 13 July 2013;

first published online 21 August 2013)

Evidence of persistent layering, with a vertical stacking of sharp variations in temperature, has been presented recently at the vertical and lateral periphery of energetic oceanic vortices through seismic imaging of the water column. The stacking has vertical scales ranging from a few metres up to $100 \mathrm{~m}$ and a lateral spatial coherence of several tens of kilometres comparable with the vortex horizontal size. Inside this layering, in situ data display a $\left[k_{h}^{-5 / 3} k_{h}^{-2}\right]$ scaling law of horizontal scales for two different quantities, temperature and a proxy for its vertical derivative, but for two different ranges of wavelengths, between 5 and $50 \mathrm{~km}$ for temperature and between $500 \mathrm{~m}$ and $5 \mathrm{~km}$ for its vertical gradient. In this study, we explore the dynamics underlying the layering formation mechanism, through the slow dynamics captured by quasi-geostrophic equations. Three-dimensional high-resolution numerical simulations of the destabilization of a lens-shaped vortex confirm that the vertical stacking of sharp jumps in density at its periphery is the three-dimensional analogue of the preferential wind-up of potential vorticity near a critical radius, a phenomenon which has been documented for barotropic vortices. For a small-Burger (flat) lens vortex, baroclinic instability ensures a sustained growth rate of sharp jumps in temperature near the critical levels of the leading unstable modes. Such results can be obtained for a background stratification which is due to temperature only and does not require the existence of salt anomalies. Aloft and beneath the vortex core, numerical simulations well reproduce the $\left[k_{h}^{-5 / 3} k_{h}^{-2}\right]$ scaling law of horizontal scales for the vertical derivative of temperature that is observed in situ inside the layering, whatever the background stratification. Such a result stems from the tracer-like behaviour of the vortex stretching component and previous studies have shown that spectra of tracer fields can be steeper than -1 , namely in $-5 / 3$ or -2 , if the advection field is very compact spatially, with a $-5 / 3$ slope corresponding to a spiral advection of the tracer. Such a scaling law could thus be of geometric origin. As for the kinetic and potential energy, the $k_{h}^{-5 / 3}$ scaling law can be reproduced numerically and is enhanced when the background stratification profile is strongly variable, involving sharp jumps in potential vorticity such as those observed in situ. This raises the possibility of another plausible mechanism leading to a $-5 / 3$ scaling law, namely surface-quasi-geostrophic

$\dagger$ Email address for correspondence: Claire.Menesguen@ifremer.fr

$\ddagger$ Professor Bach Lien Hua passed away before the final revision of this paper was completed. This manuscript was however written by the first author. 
(SQG)-like dynamics, although our set-up is more complex than the idealized SQG framework. Energy and enstrophy fluxes have been diagnosed in the numerical quasigeostrophic simulations. The results emphasize a strong production of energy in the oceanic submesoscales range and a kinetic and potential energy flux from mesoscale to submesoscales range near the critical levels. Such horizontal submesoscale production, which is correlated to the accumulation of thin vertical scales inside the layering, thus has a significant slow dynamical component, well-captured by quasi-geostrophy.

Key words: geostrophic turbulence, quasi-geostrophic flows, stratified flows

\section{Context}

Recent high-resolution observations have revealed the existence of small-scale structures in the vicinity of energetic mesoscale features. Specifically, oceanographers have introduced the terminology of 'submesoscale' to refer to the dynamical features such as filaments or fronts, in the $0.1-10 \mathrm{~km}$ range, that are smaller than mesoscale eddies (e.g. Lapeyre \& Klein 2006). Presently most of these studies on submesoscale dynamics have concerned the oceanic surface layers (upper $400 \mathrm{~m}$; e.g. Klein et al. (2008) and references therein). An exception is the quasi-geostrophic study by Smith \& Ferrari (2009) of thermohaline variance dynamics below the main oceanic thermocline, in the 800-1800 m depth range. Moreover, as developed in the following section, a new realm of unprecedented high-resolution observations using multichannel seismic reflection methods has revealed the ubiquity of fine-scale structures at the periphery of deep ocean vortices, far from the ocean surface layers.

The notable proximity in physical space of these small-scale structures to the dominant mesoscale features raises the question of energy exchanges across lateral scales of motion. More generally, it is connected to the possibility of 'a direct route to dissipation in the ocean interior by a forward energy cascade' to smaller (horizontal) scales (Molemaker, McWilliams \& Capet 2010).

The latter question is also related to the vast body of literature which attempted to find a rationale for the $-5 / 3$ spectral slope, first observed by Naström \& Gage (1985) for the atmospheric mesoscale, in terms of turbulent regimes and their associated energy cascades, and in the oceans (Riley \& Lindborg 2008, and references herein). In order to understand atmospheric turbulence at mesoscales, Waite \& Bartello (2004) and Lindborg (2005) have studied the effects of rotation on stratified turbulence and shown the existence of a critical Rossby number above which the direction of the stratified energy cascade is reversed. Indeed, Brethouwer et al. (2007) document under which conditions a $-5 / 3$ scaling law is obtained for strongly stratified turbulence and, furthermore, show that a distinctive feature is the spontaneous formation of thin horizontal layers. In addition to these somewhat idealized calculations, realistic atmospheric general circulation models have also reproduced $-5 / 3$ scaling laws (Hamilton, Takahashi \& Ohfuchi 2008). Recently, Molemaker et al. (2010) and Vallgren, Deusebio \& Lindborg (2011), in different flow geometries, have been able to numerically simulate quite extended inertial ranges in $-5 / 3$, with constant downscale energy fluxes for fully turbulent forced flows. We want to stress that in both studies a necessary condition for reproducing such inertial ranges is to involve a characteristic Rossby number significantly larger than 1 . However, note that spectral scaling laws 
provided by measurement can also be close to -2 . This spectral slope has been interpreted (Samelson \& Paulson 1988) as inertia gravity wave dynamics.

Here, we address a related energy flux question but from a deterministic point of view: how can a deep coherent vortex, such as an oceanic Meddy (see the following section), leak its energy and tracer properties to its environment through the formation of thin quasi-horizontal layers?

In the oceanic literature, stacked layers commonly observed in numerous locations of the world's oceans have been interpreted in terms of viscous/diffusive instability mechanisms, as in Ruddick \& Richards (2003) for double diffusive instability or in McIntyre (1970) for visco-diffusive instability. Furthermore, Balmforth, Smith \& Young (1998) invoked a non-monotonic relation between the buoyancy flux and the buoyancy gradient to explain the dynamics of layers and interfaces of turbulent stratified flows.

Alternately, we focus here on quasi-inviscid and quasi-non-diffusive dynamics to address the layering formation problem by choosing to study it with quasi-geostrophic dynamics, without salinity effects in the density field.

The paper is organized as follows: $\S 2$ documents the high-resolution in situ observations of small-scale structures at the periphery of an anticyclonic vortex; $\$ 3$ reports on results of quasi-geostrophic simulations which succeed in reproducing the observed layering at the periphery of a vortex; $§ 4$ discusses our results.

\section{High-resolution in situ observations of an anticyclonic eddy}

Although multichannel seismic reflection methods have been used for decades to image the solid earth beneath the seabed, Holbrook et al. (2003) only recently demonstrated the feasibility to continuously map the internal structure of the water column with an outstanding resolution of $10 \mathrm{~m}$ in both horizontal and vertical directions, using conventional towed hydrophone arrays and acoustic air gun sources.

Figure 1 shows an example of a seismic section acquired in April-May 2007 during the Geophysical Oceanography (GO) Experiment (Hobbs 2007) in the Gulf of Cadiz (North East Atlantic), synchronously and collocated with more traditional physical oceanographic data based on temperature and salinity profiles. The quantity which is displayed is the acoustic reflectivity (the stronger, the darker), induced by vertical variations of the acoustic impedance, defined as the product of the fluid density by the sound speed, which is largely controlled by temperature variations (Nandi et al. 2004), although at certain depths Sallarès et al. (2009) showed that the salinity influence may not be negligible.

The Mediterranean eddy, Meddy hereafter (Meddies are anticyclonic lens-shaped eddies that are easily recognized because they trap Mediterranean water in their core (Armi et al. 1989; Richardson, Bower \& Zenk 2000); they are particularly abundant in the Mediterranean Water outflow in the 600-1300 $\mathrm{m}$ depth range and are very long-lived vortices with a life span that can exceed 2 years, with characteristic Rossby number in the range 0.3-0.7), that appears in the centre of the figure is acoustically marked by a concentration of vertically stacked acoustic reflectors, with a typical 10-100 m vertical separation between reflectors, and a given reflector can be traced on horizontal distances that can exceed $30 \mathrm{~km}$ (Biescas et al. 2008).

What is striking in this image is the spatial concentration of acoustic reflectors, especially in the immediate vicinity of the central Meddy, with the strongest signal located directly above the eddy. These vertically stacked acoustic reflectors are wellcorrelated with vertically stacked temperature anomalies and hereafter, we will use the 


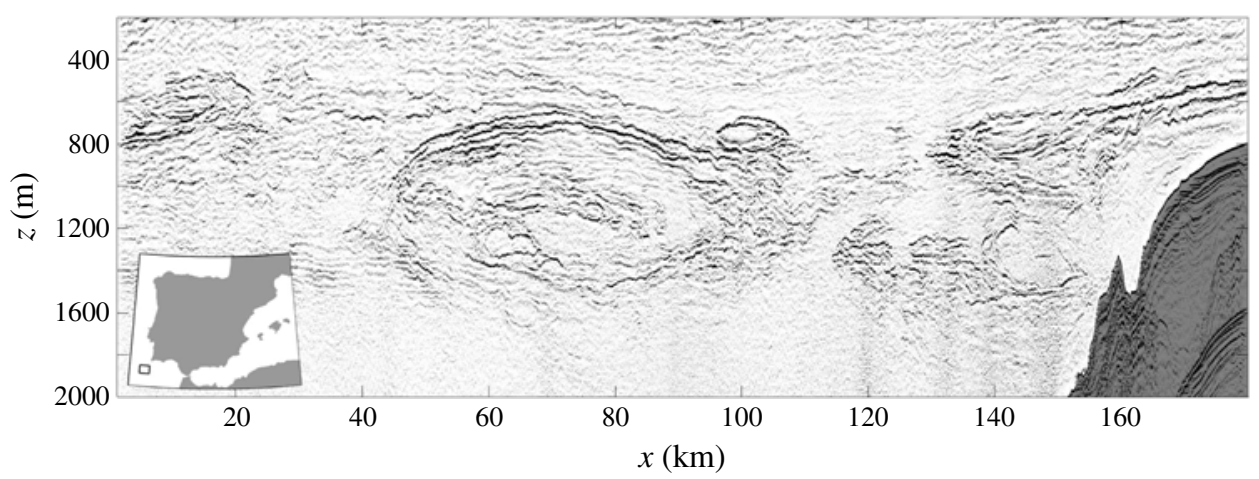

FIGURE 1. Seismic image of the internal ocean structure in the Gulf of Cadiz (right inset) during the GO experiment. The seismic layout included 6 airguns for a total volume of 2320 cubic inches, with a nominal tow depth of $11 \mathrm{~m}$ leading to a frequency band width of 5-60 Hz and a streamer with 192, $12.5 \mathrm{~m}$ spacing, seismic traces. The figure horizontal extension is $180 \mathrm{~km}$ long and it covers the 200-2000 m portion of the water column. The pre-processing of seismic data, apart from amplitude recovery and band-pass filtering, notably includes the damping of the direct wave which blurs the shallow reflectors, via a singular value decomposition filter (Golub \& Van Loan 1996). Seismic data processing is limited to constant velocity dynamic corrections (normal move out) and summation (stack).

terminology of 'layering' to refer to such phenomenon, taking into account their large lateral extension. The existence of vertically stacked anomalies surrounding anticyclonic eddies had previously been shown using data from conventional Expendable Bathythermograph (XBT) temperature profiles (Pingree \& Le Cann 1993). More generally, acoustically marked 'layering' appears to occur in the proximity of energetic mesoscale features.

In the case of acoustic images, either internal waves (e.g. Gonella \& Michon 1988; Holbrook \& Fer 2005) or double-diffusive processes (Biescas et al. 2008) have been invoked to explain the observed layering. An alternative viewpoint has been proposed by Ménesguen et al. (2009): taking advantage of the fine resolution of long seismic transects, oceanic turbulence properties over several decades of horizontal scales have been characterized using migrated acoustic reflectivity data. A horizontal scaling law in $-5 / 3$ is found to extend from $100 \mathrm{~m}$ to $4 \mathrm{~km}$ horizontal wavelength for the most intense acoustic reflectors which surround strong anticyclonic eddies.

Here, we revisit a part of the GO dataset in order to obtain an acoustic reflectivity signal. The seismic data treatment is the conventional one, but with a processing bin enlarged to $50 \mathrm{~m}$ in order to reduce the signal-to-noise ratio. The seismic instrument (e.g. source) filter has been removed through deconvolution (figure $2 a$ ). The source signature being unavailable, it is estimated from the near-offset sea-bottom reflections. However, the seismic data content remains mainly driven by the source frequency bandwidth, which, in our study, lies between 20 and $60 \mathrm{~Hz}$. As was shown in Ménesguen et al. (2009), the range of horizontal scales actually probed by different acoustic sources is found to be directly linked to their frequency content. Here, an estimation of the well-represented horizontal scales is between $500 \mathrm{~m}$ and $5 \mathrm{~km}$.

We emphasize that the deconvoluted acoustic reflectivity is more precisely controlled by the vertical derivative of temperature, filtered by the seismic source bandwidth. Therefore, acoustic reflectivity is proportional to a filtered quasi-geostrophic vortex 

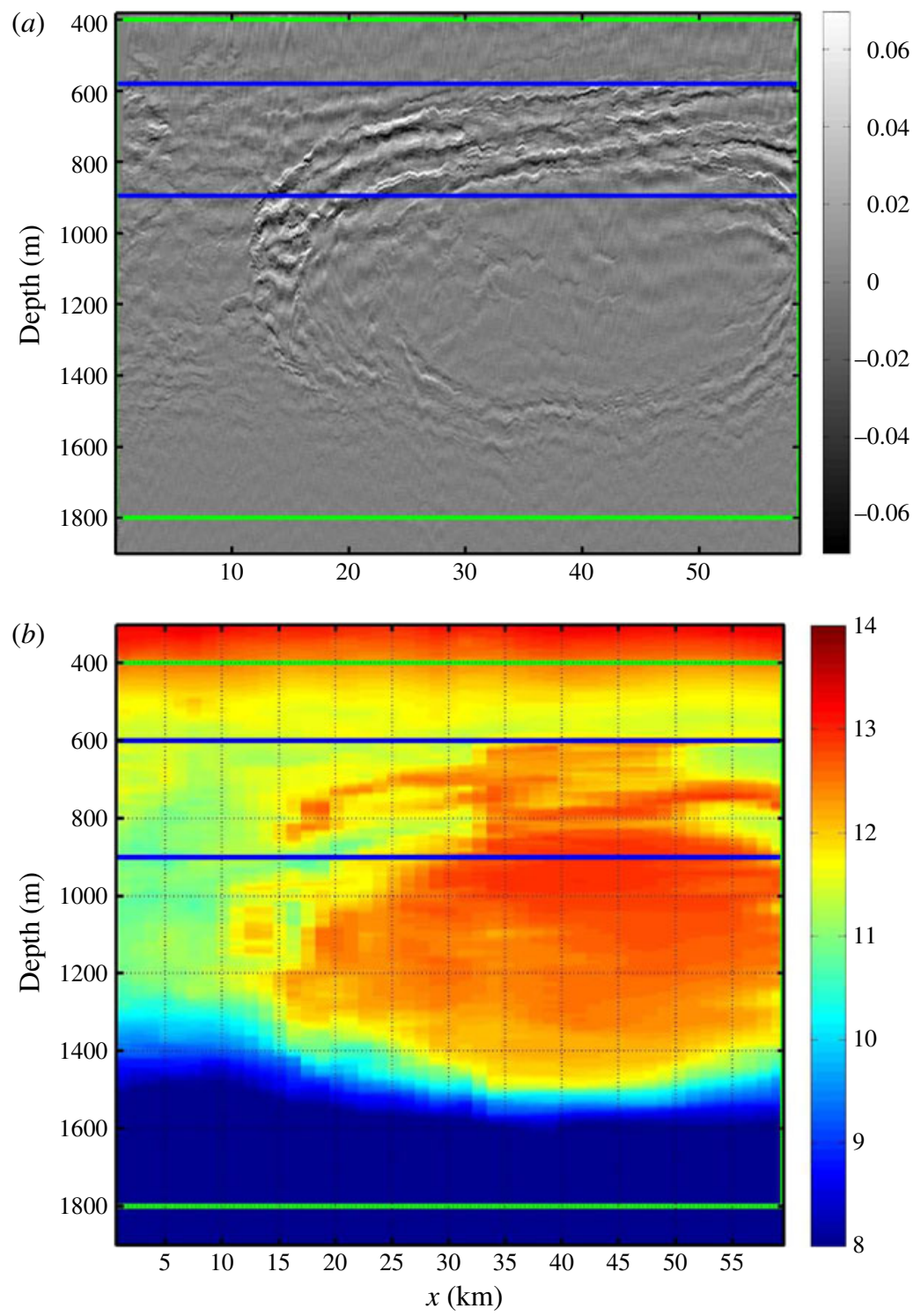

FIGURE 2. (a) Vertical section of the deconvoluted acoustic reflectivity across the same Meddy as in figure 1 but for a different transect. (b) Synchronous temperature field across the Meddy from the colocated XBT survey with a grid spacing of 2-3 km.

stretching: this will be the cornerstone of the quasi-geostrophic modelling presented in the next section which provides a rationale for layering formation.

During the GO experiment, concomitantly to the seismic measurements, XBT temperature profiles were taken at regular intervals. In particular, the section corresponding to figure 2 was sampled synchronously by seismic methods and by an XBT survey with a 2-3 $\mathrm{km}$ grid sampling.

Results are presented in figure 3 in terms of horizontal wavenumber spectra, the blue and green curves corresponding to the blue and green boxes, respectively, for 
(a)

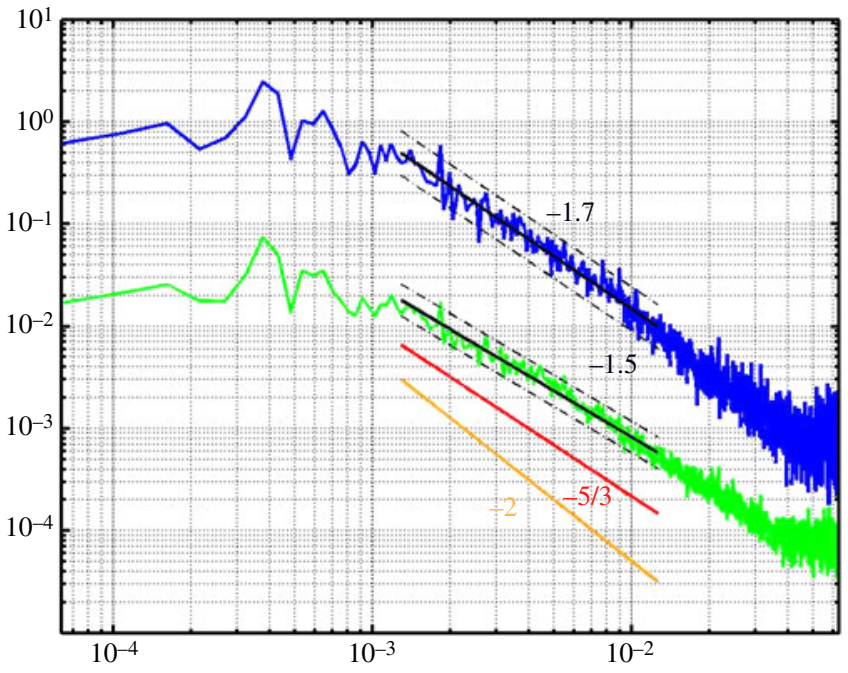

(b)

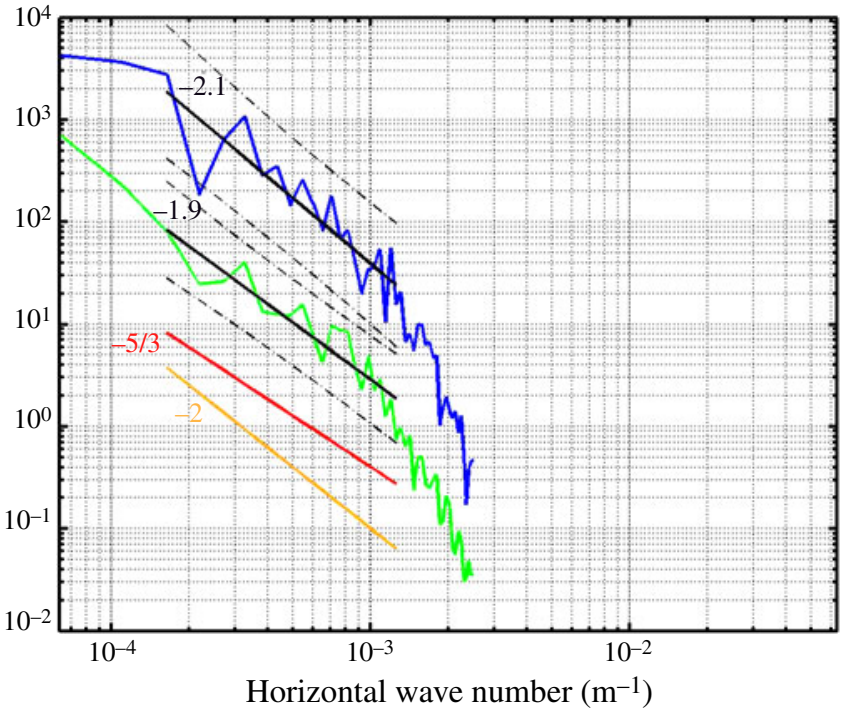

FIGURE 3. (a) Horizontal wavenumber spectra of acoustic reflectivity in the two boxes of figure 2(a). (b) Horizontal wavenumber spectra of temperature in the two boxes of figure 2(b). Blue (respectively, green) curves correspond to the blue (respectively, green) box of figure 2 . Green curves have been shifted by one decade for better reading. Continuous black lines correspond to the best linear fit of the spectra slopes, with an error estimate corresponding to the dotted lines.

seismic data (figure $3 a$ ) and for XBT temperature signal (figure $3 b$ ). The horizontal axis corresponds to horizontal wavenumbers for both figures, and clearly reflects the differences in resolution for the two types of acquisition. The most important result is the existence of scaling laws over one decade of scales in both data sets.

In the layering above the Meddy, acoustic reflectivity displays a $k_{h}^{-5 / 3}$ range for wavelengths comprised between $500 \mathrm{~m}$ and $5 \mathrm{~km}$. Such characteristics of the acoustic 


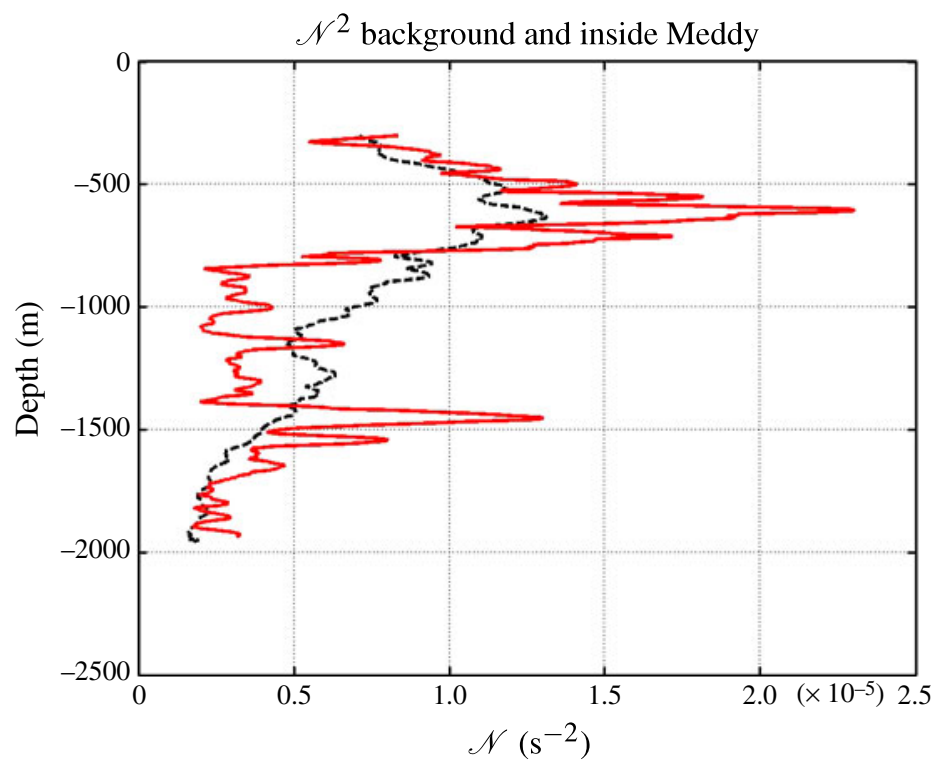

FIgURE 4. (Colour online) Vertical profiles of $\mathscr{N}^{2}$ measured during the GO experiment. The black dashed curve corresponds to the background stratification obtained by averaging 14 vertical profiles located outside the Meddy, while the continuous curve corresponds to a vertical profile located inside the Meddy core.

reflectivity spectrum holds in both the blue box, between 600 and $900 \mathrm{~m}$ depth, which contains the most intense reflectors, but also in the larger size green box. In the latter case, the signal variance is clearly dominated mostly by the variance of the upper layering signal.

As for the temperature signal of figure $3(b)$, horizontal spectral slopes between $-5 / 3$ and -2 are present for horizontal scales between 5 and $50 \mathrm{~km}$. Such a range is limited, on one side, by the much coarser horizontal grid of the XBT survey and, on the other side, by the finite lateral extension of the layering of figure 2(b). Such spectra were also measured in Samelson \& Paulson (1988) who had linked the spectral slopes to a signature of inertia gravity waves.

Both figure 3(a) and $(b)$ contain scaling laws comprised between $-5 / 3$ and -2 , but for two different physical quantities (acoustic reflectivity and temperature, respectively) and for different ranges of scales. Both types of measurements are thus complementary in spectral space, a property which has already been used by Papenberg et al. (2010) in their high-resolution temperature two-dimensional section reconstruction.

During the GO experiment, CTD (conductivity, temperature, depth) casts were also available and figure 4 documents vertical profiles of Brunt-Väisälä frequency $(\mathscr{N})$. The black curve corresponds to the background stratification obtained by averaging 14 vertical profiles located outside the Meddy, while the red curve corresponds to a vertical profile located inside the Meddy core. The eddy core, between 800 and $1400 \mathrm{~m}$ depth, is characterized by an almost constant $\mathscr{N}^{2}$, while upper and lower boundaries act as barriers with strong positive deviations of $\mathscr{N}^{2}$. The background stratification displays a marked thermocline around $700 \mathrm{~m}$ depth, with a secondary weaker thermocline around $1300 \mathrm{~m}$ depth. Both are characteristics of the double climatological thermocline of the Gulf of Cadiz. 
Finally, additional concomitant physical oceanography measurements led to a rough estimate of the Rossby number of the observed Meddy to be $\sim 0.25$ and therefore far from an inertially unstable regime.

\section{Quasi-geostrophic simulations}

Observations presented in the previous section raised the following questions.

(a) What is the underlying dynamics leading to the formation of the layering observed at the periphery of the Meddy, mostly aloft and beneath such an anticyclonic vortex?

(b) More generally, can the small scales associated with the layering, that typically lie in the so-called submesoscale range, be created from the mesoscale structure of the Meddy?

(c) Can the scaling laws between $-5 / 3$ and -2 , that are seen both in the vertical derivative of temperature (as seen in acoustic reflectivity) and in temperature, be reproduced using the slow dynamics of quasi-geostrophic equations?

\subsection{Initial base state and background stratification}

In order to answer such questions, we have performed highly idealized simulations of the dynamics of an anticyclonic eddy in a rotating stratified flow on an $f$-plane, where $f$ is the constant Coriolis parameter, using quasi-geostrophic equations.

The conservation of quasi-geostrophic potential vorticity (PV) reads (Pedlosky 1996)

$$
\frac{\partial Q}{\partial t}+J(\Psi, Q)=v \nabla^{6} Q
$$

where $\Psi$ is the streamfunction, $J(\cdot, \cdot)$ the horizontal Jacobian operator and $Q$ the PV defined as

$$
Q \equiv \nabla^{2} \Psi+\left(\frac{f^{2}}{\mathscr{N}^{2}} \Psi_{z}\right)_{z}+f
$$

where $\nabla \equiv(\partial / \partial x, \partial / \partial y), z$ is the vertical coordinate, $f$ is the Coriolis parameter and $\mathscr{N}$ is the background Brunt-Väisälä profile and $v$ is hyperviscosity required to dissipate potential enstrophy at the smallest scales in the numerical simulations.

Lateral and vertical characteristic scales of the vortex are denoted as $L$ and $H$, respectively. The absolute value of the relative vorticity at the vortex centre is $\zeta_{0}$. They are used as characteristic values for the non-dimensionalization:

$$
\tilde{r}=\frac{r}{L}, \quad \tilde{z}=\frac{z}{H}, \quad \tilde{\psi}=\frac{\psi}{\zeta_{0} L^{2}}, \quad \tilde{t}=\zeta_{0} t .
$$

Hereafter, we drop the . Specifically, the compact support function which is used to represent the vortex lens is

$$
\begin{aligned}
\bar{\psi}(r, z) & =\frac{1}{4 \alpha} \frac{1}{\left(1+d^{2}+d^{4}\right)^{\alpha}}, \\
d^{2} & \equiv r^{2}+\frac{\mathscr{N}^{2}}{\mathscr{N}_{0}^{2}} z^{2},
\end{aligned}
$$

with $\alpha$ chosen to be 1 or 2 . Taylor expansions near the vortex centre gives respectively a $r^{2}$ and a $r^{4}$ radial dependence of the PV field associated with the streamfunction in (3.4), yielding a base state with a nearly homogenized PV near the vortex centre, a 
property shared by many geophysical vortices. Note that the sign of (3.4) corresponds to the case of an anticyclonic vortex core, since we want to model the evolution of a Meddy: however, this has no dynamical consequences in the quasi-geostrophic framework which is invariant under reversal of PV sign. Here $\mathscr{N}(z)$ is chosen either constant or depth-variable, while $\mathscr{N}_{0}$ is a characteristic value of the background stratification. The Burger number of the base state is

$$
B u \equiv \frac{\mathscr{N}_{0}^{2} H^{2}}{f^{2} L^{2}} .
$$

In all of the following simulations, the Burger number, the characteristic value of the background stratification and the lateral characteristic scale $L$ have been specified and the scaling height $H$ is deduced.

The PV anomaly $\bar{Q}$ of the base state is

$$
\bar{Q}(r, z)=\left(r \bar{\psi}_{r}\right)_{r} / r+B u^{-1}\left(\frac{\mathscr{N}_{0}^{2}}{\mathscr{N}^{2}} \bar{\psi}_{z}\right)_{z} .
$$

Two simulations, hereafter referred to as runs A and B, corresponding to constant (red curves in figure 5) or depth-variable $\mathscr{N}^{2}(z)$ (blue curves in figure 5), respectively, will be analysed in the rest of this section. Figure 5(a) displays the background stratification outside the Meddy, while the black curve in figure 5(b) displays the in situ Meddy-induced anomaly in Brunt-Väisälä frequency, dimensionally, $\delta \mathscr{N}^{2} \equiv f \bar{\psi}_{z z}$, and the red and blue curves are fits of the simulated basic state using (3.4). The base state defined by (3.4) differs from the Gaussian streamfunction used in Nguyen et al. (2012, hereafter NHSC12) which implied an excessively large relative amplitude of negative stretching over the positive stretching above and below the eddy core, when compared with the $\mathscr{N}^{2}(z)$ barriers at the upper and lower boundaries of the Meddy that are evidenced in figure 4. Furthermore as evidenced by figure 5, in the case of variable $\mathscr{N}(z)$, the shape of (3.4) is particularly appropriate to fit the observed profile.

The numerical experiments reported here correspond to spin-down experiments for a flat lens vortex with small Burger number $(B u<0.5)$ and a moderate initial relative vorticity at the vortex centre, as observed for the GO experiment Meddy. The background stratification is assumed to be due to temperature only and any effect due to salinity is discarded, thus preventing the occurrence of double diffusion. Both uniform and vertically varying Brunt-Väisälä profiles will be considered for regimes of strongly stratified fluid with values of $\mathscr{N}_{0} / f \approx 20-40$.

The numerical code is the spectral three-dimensional quasi-geostrophic code of Hua \& Haidvogel (1986) which has been parallelized to run on the Earth Simulator 2 (see Appendix). Typical resolutions correspond to resolutions of $1000^{2} \times 600$ grid points, corresponding to a horizontal grid size of $\mathrm{d} x=200 \mathrm{~m}$ and a vertical grid size of $\mathrm{d} z=4 \mathrm{~m}$.

\subsection{Critical-level baroclinic instability}

The slow quasi-geostrophic unstable modes of a lens vortex in a continuously stratified flow studied in NHSC12 were found to correspond to the group of low azimuthal modes $m=1,2,3,4,5$. Their relative importance mainly depends on the aspect ratio of the lens, and hence its Burger number. For $B u<1$ (cf. figure $1 c$ in NHSC12), higher azimuthal modes are favoured for small-aspect-ratio vortices. Here $B u<1$ corresponds to a regime where vortices destabilize mostly through baroclinic instability, with typical growth rates significantly slower than the vortex rotation period 
(a)

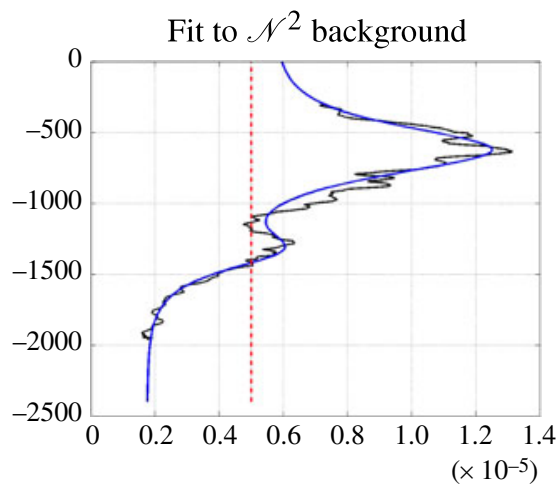

(b)

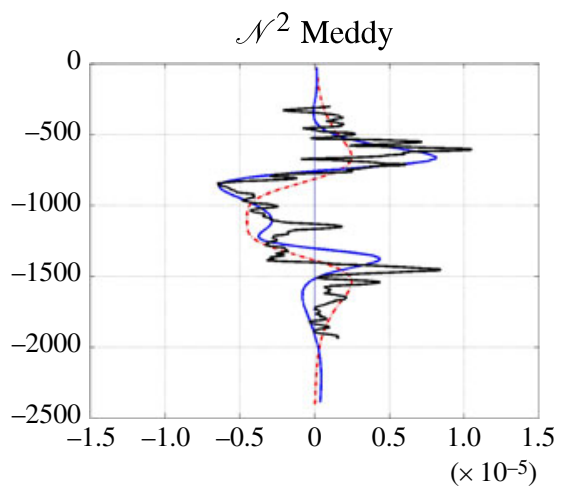

FIGURE 5. (a) Background stratification profiles: the black line corresponds to the observed background stratification of figure 4. The constant, $\mathscr{N}_{0}^{2}$, and depth-variable, $\mathscr{N}^{2}(z)$, stratification profiles used in the numerical simulations respectively correspond to the red dashed-dotted and blue continuous lines. (b) Vertical profiles of the eddy-induced anomaly in Brunt-Väisälä frequency, dimensionally, $\delta \mathscr{N}^{2} \equiv f \bar{\psi}_{z z}$, for the basic states defined by (3.4) for constant (red dash-dotted line, run A) and depth-variable (blue continuous line, run B) background stratification. Characteristics scales are $L=32 \mathrm{~km}, f=8 \times 10^{-5} \mathrm{~s}^{-1}$ and $\mathscr{N}_{0}^{2}=5 \times 10^{-6} \mathrm{~s}^{-2}$ (dashed-dotted red line of figure $5 a$ ). For run A: $B u=0.15, \zeta_{0}=0.3 f$; for run $\mathrm{B}: B u=0.4, \zeta_{0}=0.5 f$. The black continuous line corresponds to the in situ eddy-induced anomaly of figure 4 .

for moderate-amplitude flows. This predominance of baroclinic instability for 'flat' vortices is to be contrasted to the regime of predominant barotropic instability of 'tall' vortices (Gent \& McWilliams 1986; Dritschel \& de la Torre Juarez 1996). Baroclinic instability is associated with a critical level which is located at the vortex periphery (see figure 4 of NHSC12). As elaborated on in NHSC12, this critical level can be identified as being an extremum of the disturbance PV. It can be evidenced by a forward time integration of the PV equation linearized around the basic state defined by (3.4) and initialized with random noise.

For a constant background stratification (run A), the results (not shown) are similar to those documented in NHSC12 for the case of a Gaussian streamfunction vortex. For run B, corresponding to the depth-variable background stratification of figure 5(a), figure $6(a, b)$ show the horizontal and vertical sections of the disturbance PV respectively at a depth of $750 \mathrm{~m}$ and through the vertical axis of symmetry of the vortex, after 120 days of integration. The horizontal section figure 6(a) shows the dominance of both an azimuthal mode $m=1$ entirely located inside the eddy core and a mode $m=2$ which is instead well outside the eddy core. The vertical section figure $6(b)$ shows the depth localization of the critical levels of both modes, with mode $m=2$ located further away from the eddy centre than mode $m=1$. Both modes have growth rates of comparable amplitude, with a slight dominance of mode $m=1$ that can be evidenced by pursuing the integration of the linearized PV equation much longer in time (not shown).

A notable result is the quite strong spatial concentration of the PV disturbance both in lateral and vertical coordinates, leading to very sharp 'jump-like' variations of the PV field. Moreover, while quasi-geostrophic PV is the sum of the relative vorticity and vortex stretching terms, the simulations show that, in the vicinity of the critical levels, PV is completely dominated by the vortex stretching term, that is proportional 

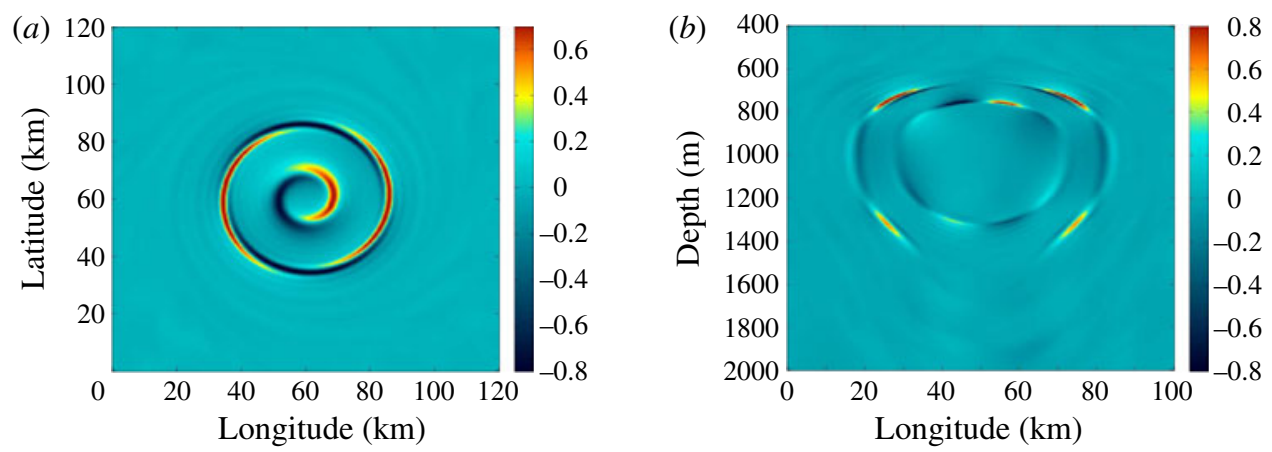

FIGURE 6. PV disturbance field: position of the critical level obtained by the forward time integration of linearized PV for run B for a depth-variable background stratification and a base state given by (3.4) for $L=32 \mathrm{~km}, B u=0.15, \zeta_{0}=0.5 f, \mathscr{N}_{0}^{2}=5 \times 10^{-6} \mathrm{~s}^{-2}$. (a) Horizontal section; $(b)$ vertical section. The colourbar is in units of $f$.
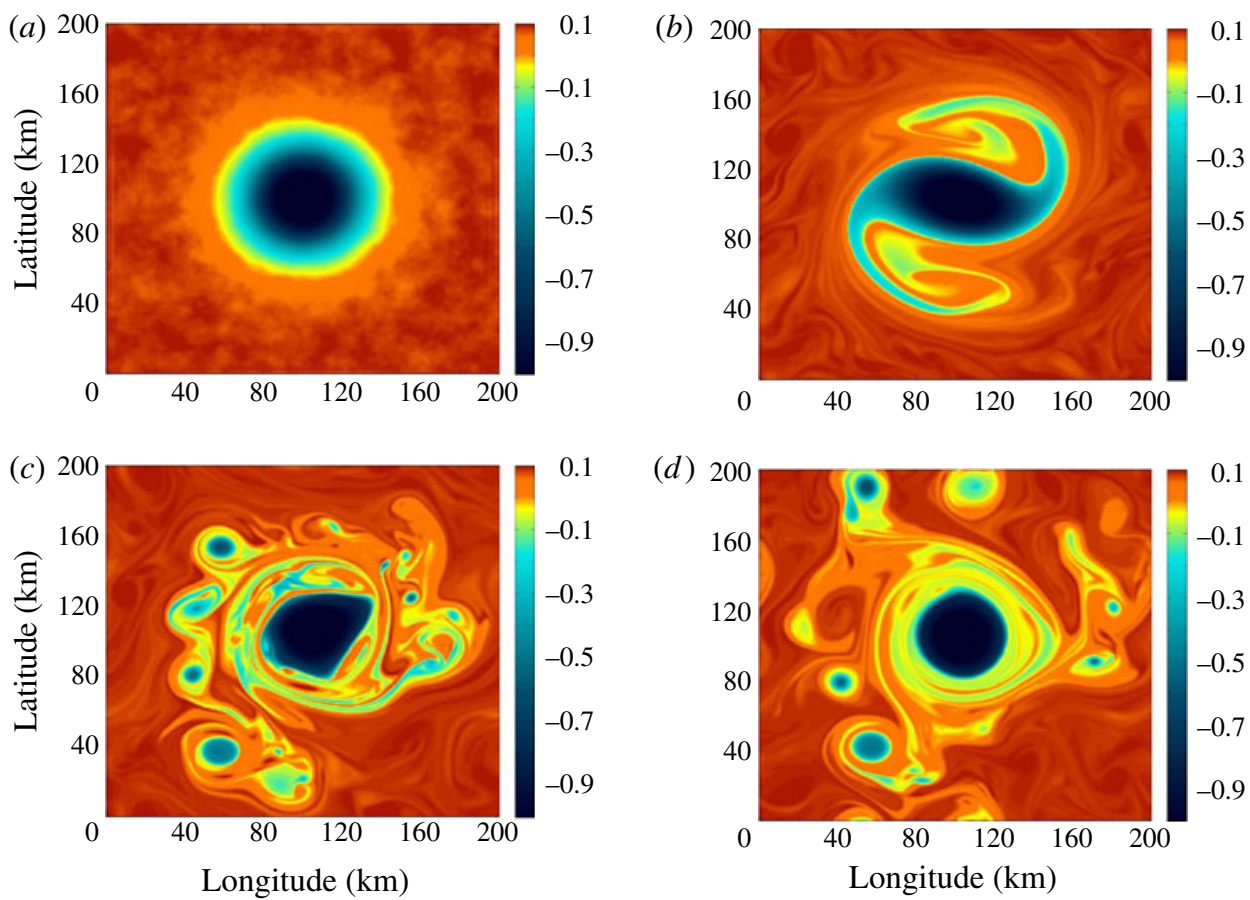

FIGURE 7. Horizontal section of PV anomaly through mid-height of the domain at $(a)$ day 0 , $(b)$ day $60,(c)$ day 120 and $(d)$ day 240 for the full nonlinear quasi-geostrophic simulation of run $\mathrm{A}$. The colourbar is in units of $f$.

to the vertical derivative of temperature, i.e. precisely to the signal that determines the acoustic reflectivity measured by seismic reflection technique.

The above linearized results have been used as an interpretation guide for the nonlinear simulations. The time integration of the fully nonlinear PV equation is performed, using as initial conditions the compact anticyclonic vortex of (3.4) with random barotropic noise and thus no a priori baroclinic scale in initial disturbances. For run A (constant background stratification), figure 7(a) shows the full initial PV 

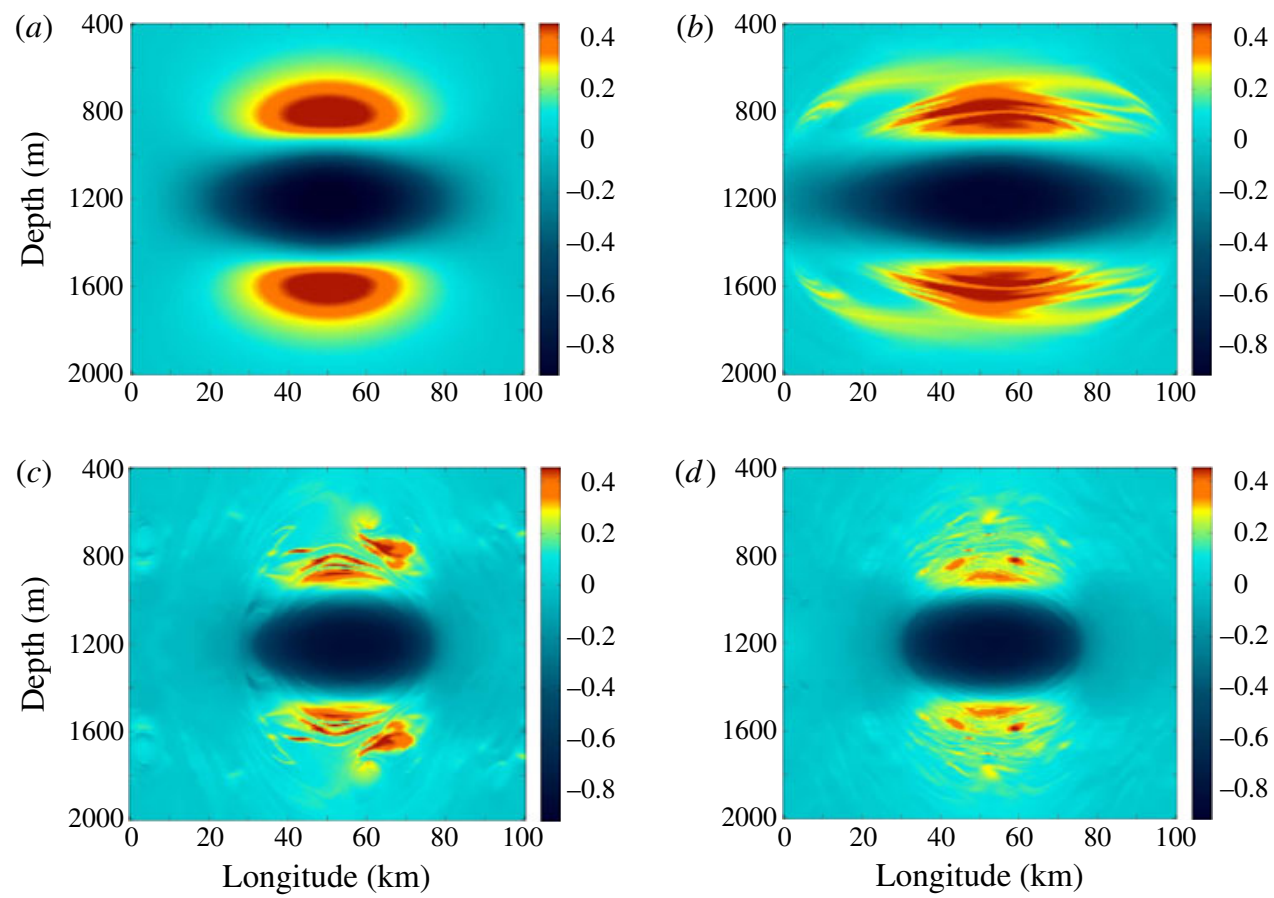

FIGURE 8. Vertical sections of vortex stretching field through the vortex axis at $(a)$ day $0,(b)$ day $60,(c)$ day 120 and $(d)$ day 240 for the full nonlinear quasi-geostrophic simulation of run A. The colourbar is in units of $f$.

at the depth of the eddy centre $(z=1100 \mathrm{~m})$. The finite-amplitude growth of modes $m=2$ (and also $m=1$ ) leads to the formation of two slightly asymmetric satellites (figure $7 b$ ). Such satellites connected by spiral arms to the main eddy core have been observed in situ in the seismic data for another Meddy structure (Ménesguen et al. 2012). A subsequent ejection of a long PV filament which destabilizes into rolls that coalesce into smaller size vortices is seen in figure $7(c)$, while the vortex core undergoes polygonal deformations due to azimuthal modes $3,4,5$ of slower growth rate. The final stage corresponds to a tendency for axisymmetrization of the central vortex core (figure $7 d$ ). The same type of phenomenology is observed in run B (not shown).

\subsection{Layering formation}

In this subsection, an overview of the phenomenology of the destabilization of the lens vortex is provided. Moreover, we will predominantly focus on vortex stretching, since it is the dominant component of PV near the critical levels above and below the vortex core and is furthermore directly related to acoustic reflectivity.

Vortex stretching changes sign between the vortex core and its upper/lower lobes. For an anticyclone, the central part of the eddy undergoes a stretching of the isotherms, while the top and bottom lobes undergo a pinching: this pinching, or positive vortex stretching, is colour coded in orange brown in figure 8 . This is where stability studies predict that the critical levels are located for a compact vortex.

In figure $8(a)$, the initial state is such that there is no disturbance in vortex stretching since initial random disturbances are purely barotropic. Figure $8(b)$ shows the transient growth of small-vertical-scale vortex stretching in the positive lobe 


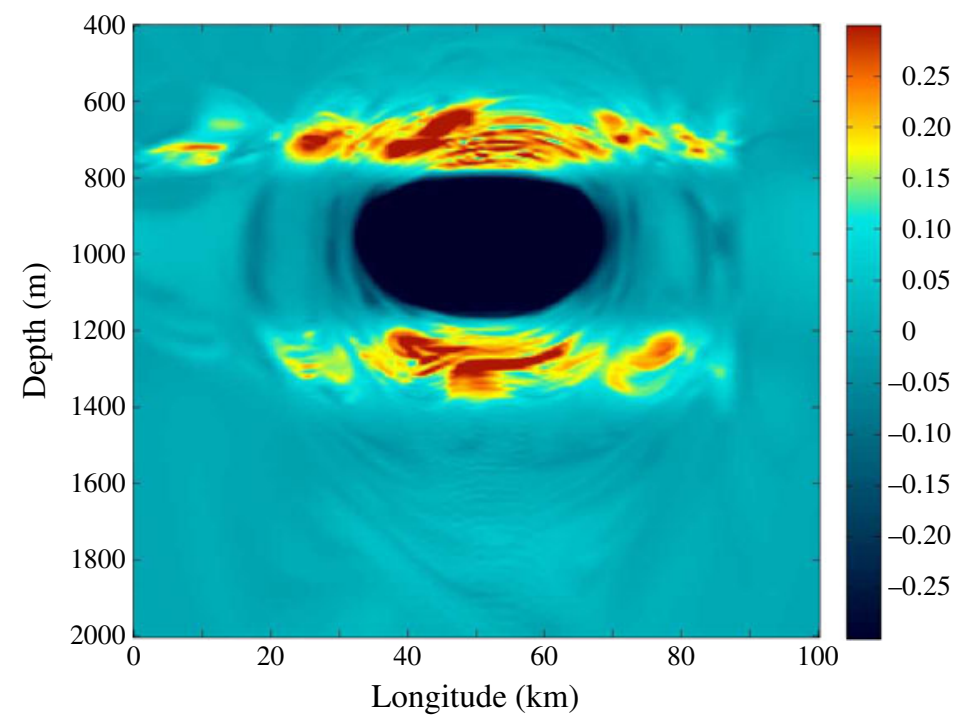

FIGURE 9. Vertical section of vortex stretching field through the vortex axis for day 210 for the full nonlinear quasi-geostrophic simulation for variable stratification case (run B). The colourbar is in units of $f$.

at day 60. Figure $8(b)$ corresponds to the finite-amplitude stage of the $m=2$ satellites and presents some resemblance to the two 'arms-like' filaments seen in the temperature field of the GO data set (figure $2 b$ ). Figures $8(d)$ and 9 demonstrate both the formation and the very long persistence of the 'layering' over the entire length of the integration, with a localization of the layering in the immediate vicinity of the critical levels found by integration of the linearized PV equation. The height of the vortex core is seen to decrease with time as more and more PV is peeled out of the central vortex core. Such results are also observed in run B, for a variable background stratification, in the vertical section of the vortex stretching field through the vertical axis of symmetry of the vortex (figure 9). An intercomparison of the latter figure with figure $6(b)$ confirms that layering remains concentrated near the critical levels of the dominant linearly unstable modes.

The above simulated phenomenology, which is very robust and occurs even for a constant background Brunt-Väisälä frequency, is a three-dimensional analogue of the preferential wind-up of PV near a critical radius, a phenomenon which has been documented for barotropic vortices by Balmforth, Smith \& Young (2001) for the case of neutral modes. An important difference here is that we are dealing with unstable modes associated with a critical-level instability. In other words, 'layering' results from the wind-up of vortex stretching near a critical level. A three-dimensional perspective of isosurfaces of vortex stretching is presented in figure 10 for run A, illustrating the helical structure of layering aloft and beneath the eddy core. Layering is thus not a simple stacking of pancake-like structures, but has a more complex three-dimensional structure.

Returning to the in situ observations, acoustic reflectivity is determined by the vertical derivative of temperature. It is furthermore set by the frequency bandwidth of the seismic instrumentation, and acoustic reflectivity is limited to a given bandwidth of vertical scales. In order to compare our simulation results with the observed acoustic 


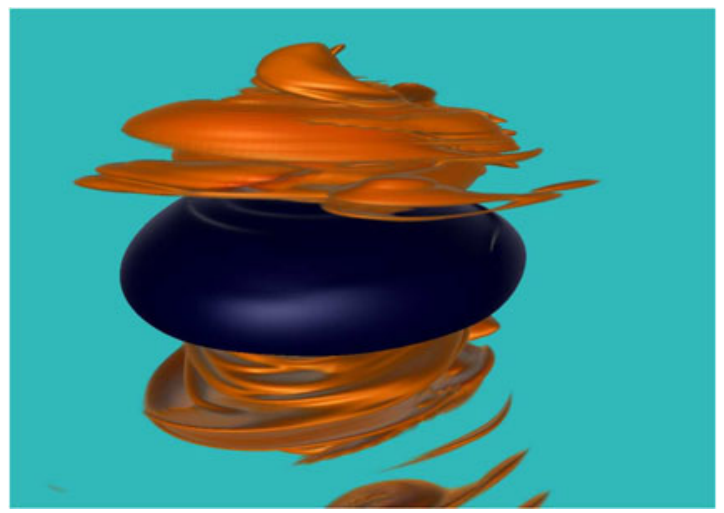

FIGURE 10. Three-dimensional perspective of vortex stretching at day 200 of run A: the blue isosurface corresponds to non-dimensional value of -0.4 and the orange isosurface to a non-dimensional value of +0.3 .

reflectivity, the vortex stretching field of figure $8(d)$ is filtered to keep the same vertical scales as those the seismic instrumentation of figure $2(a)$. It is plotted in figure 11. There are obvious resemblances in both the patterns and in the localization in physical space of the layering seen in figure $2(a)$. This supports the rationale that layering formation occurs through the slow (quasi-geostrophic) wind-up of PV jumps associated to the critical levels of baroclinic instability of the flat lens vortex.

Note in figure 11 the presence of quite tilted reflectors, above and beneath the vortex core, that are also seen in the high-resolution seismics of Geli et al. (2009) and Pinheiro et al. (2010). Analogous numerical results concerning tilted patterns are reported by Smith \& Ferrari (2009) who advocate an $f / \mathscr{N}$ slope. Figure 11 shows however a whole range of slopes for the tilted reflectors with a maximum slope close to $f / \mathscr{N}$ for a few reflectors.

Having provided evidence that the layering is attached to special depths, close to the critical levels predicted by linear theory, we now document the specific geometric patterns of layering in the horizontal plane. Figure 12 shows the horizontal patterns involved in layering formation for run A near the critical level $(z=800 \mathrm{~m})$. The initial round blob of positive vortex stretching is being wound up into a spiral shaped structure. The later stages of the simulation show that the filamentation process remains trapped right at the centre of the image. It has to be contrasted to the behaviour of PV at mid-depth in figure 7, which is mostly expelled into filaments at the lateral rim of the vortex core. Similar patterns are observed for run B.

We stress that the phenomenology is not just a simple quasi-geostrophic stirring of a tracer field, because it involves critical level instabilities, with a constant forcing of PV disturbance due to baroclinic instability and we thus expect different scaling laws from the classical $k_{h}^{-1}$ spectrum in quasi-geostrophic turbulence. Indeed, at the critical level, we are dealing with a very compact advective field, with anomalies which remain trapped near the eddy core. This situation is akin to the spiral advection of a tracer field by a compact vortex, studied by Gilbert (1988). This is further elaborated in the next subsection.

In order to quantitatively assess the tracer-like behaviour of vortex stretching near the critical levels, the stirring by the compact vortex lens of a passive tracer field with an initial random barotropic distribution has been investigated for the case of 


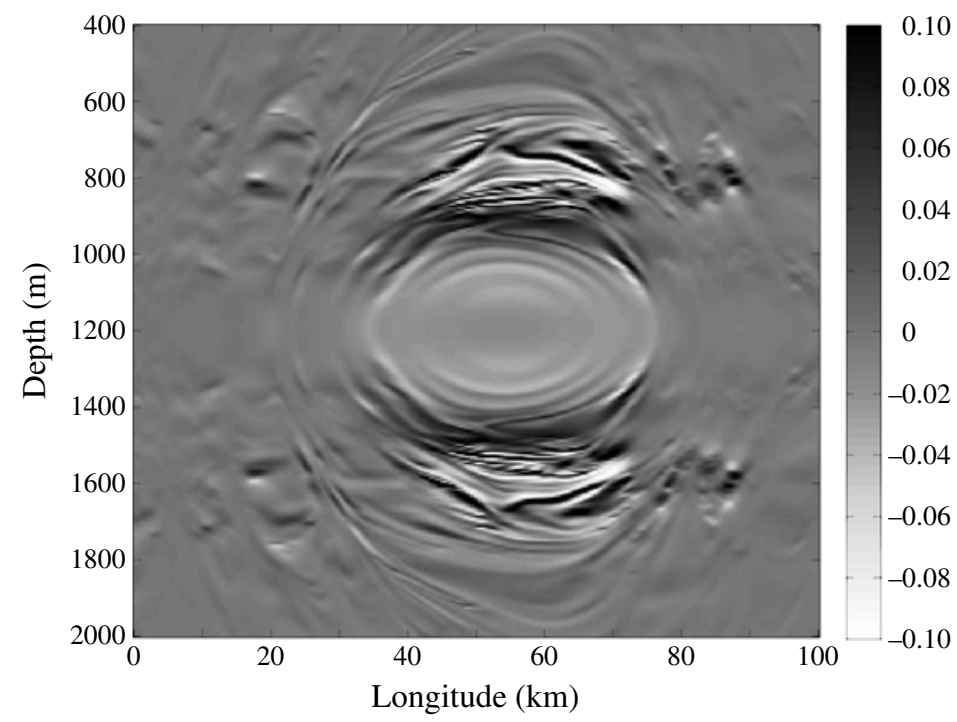

FIGURE 11. High-pass filtered acoustic reflectivity (same vertical scales as seismic acoustic source) corresponding to the vortex stretching field of figure $8(d)$ of run A.

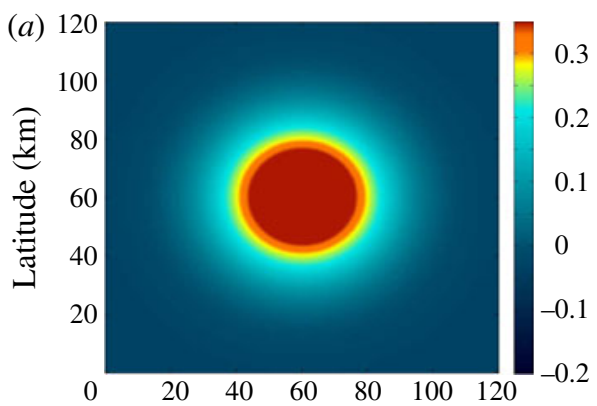

(b)
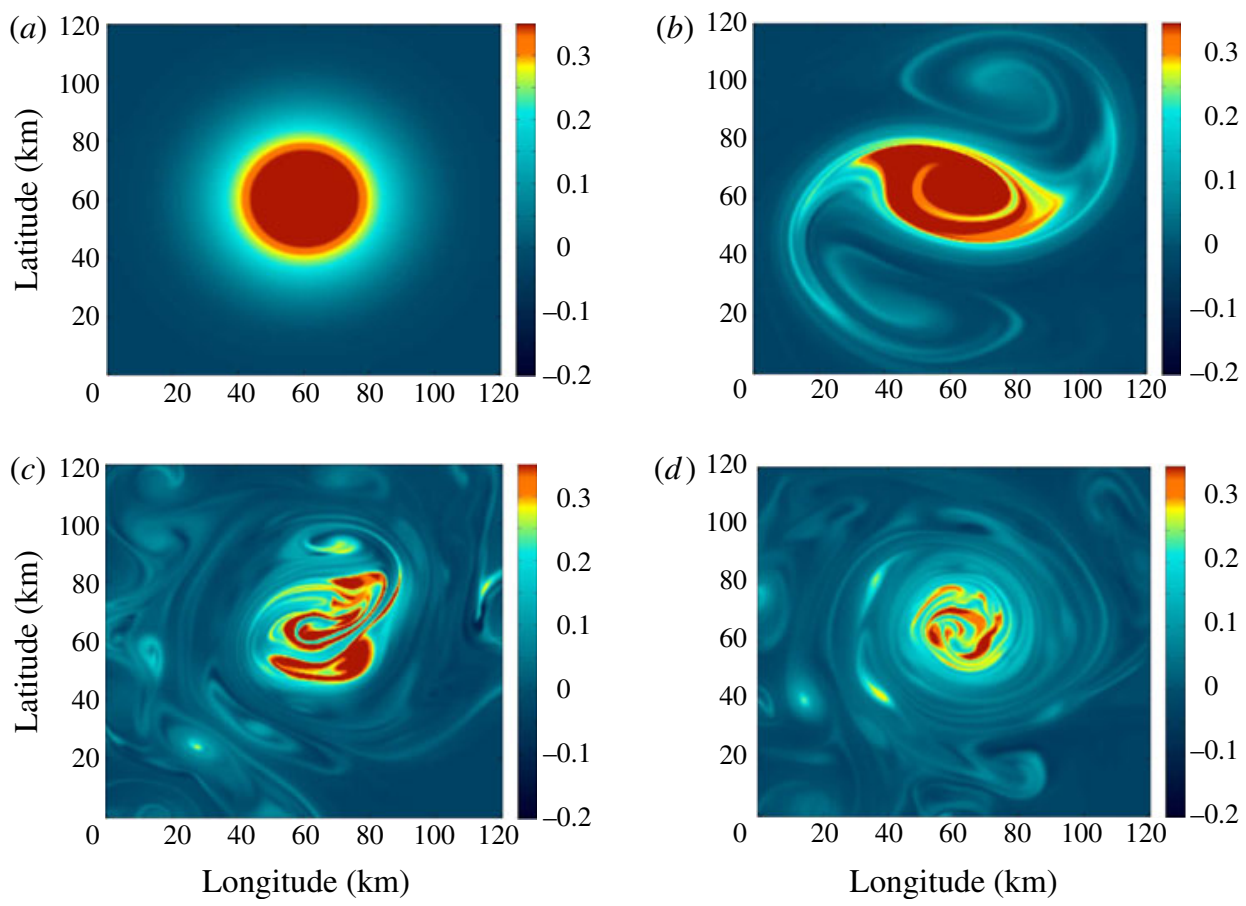

FIGURE 12. $(a-d)$ Horizontal sections of vortex stretching at the critical level of the initial vortex baroclinic instability at $(a)$ day $0,(b)$ day $60,(c)$ day 120 and $(d)$ day 240 for the full nonlinear quasi-geostrophic simulation of run $\mathrm{A}$. The colourbar is in units of $f$. 

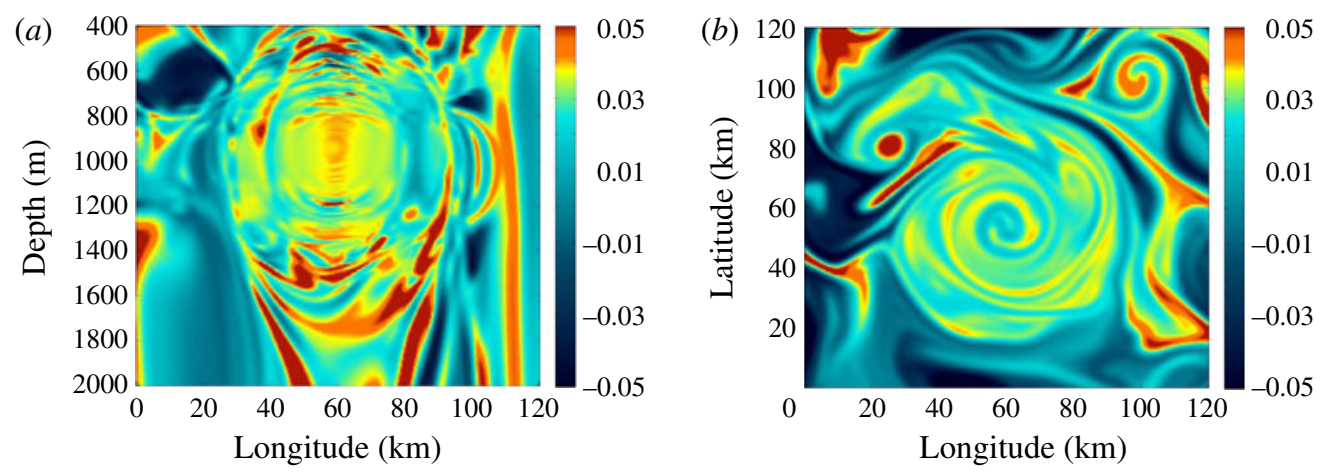

FIGURE 13. Vertical section at mid-latitude $(a)$ and horizontal section at the critical level $(b)$ of the tracer field for the variable stratification (run B).

(a)

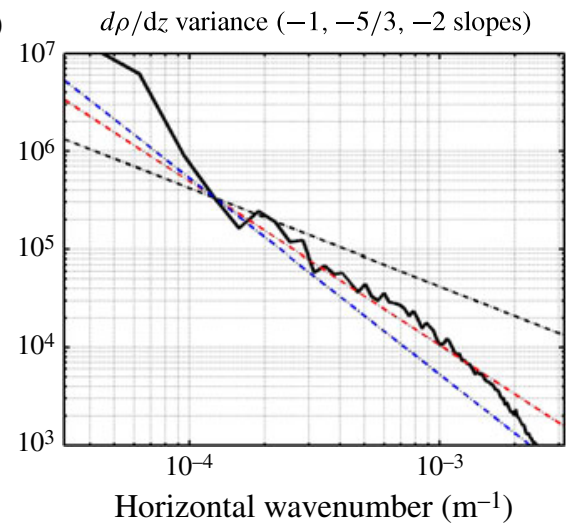

(b)

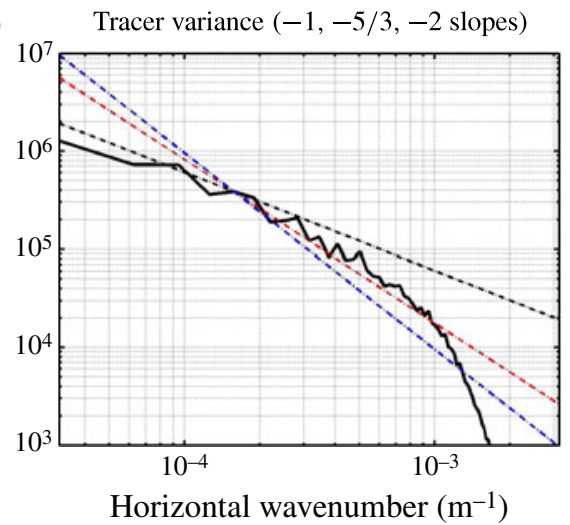

FIGURE 14. (Colour online) Run B (variable stratification): horizontal wavenumber spectra averaged around the critical level over a depth of $100 \mathrm{~m}$ of $(a)$ the vertical derivative of density and $(b)$ a tracer field. (The $y$-axes are adimensional, the $x$-axes are rescaled to match observations. These scales apply to all subsequent spectra.)

a variable background stratification (run B). The vertical section at midlatitude and horizontal section near the critical level of the tracer field are shown in figure 13(a,b), respectively, revealing similar patterns to those seen in vortex stretching (figures 8 and 12).

\subsection{Scaling laws and fluxes of energy and enstrophy}

\subsubsection{Passive tracer and vortex stretching scaling laws}

As in the in situ data (figure 3), horizontal spectral slopes in $-5 / 3$ for the vertical derivative of density, are found in run B (figure 14a) near the critical level. The range of horizontal scales involved in the $-5 / 3$ range is somewhat narrower than in the observations and slightly shifted, covering the $4-70 \mathrm{~km}$ range. We suspect that reaching the resolution of the seismic data in the numerical simulations would enlarge the ranges of the scaling laws. Note the close analogy between the power density spectra of the vertical derivative of density and of the passive tracer in figure 14(a,b), which both display a slope close to $-5 / 3$ over more than a decade of horizontal wavenumbers. The above scaling laws are reminiscent of the results of Saffman (1971) 
(a)

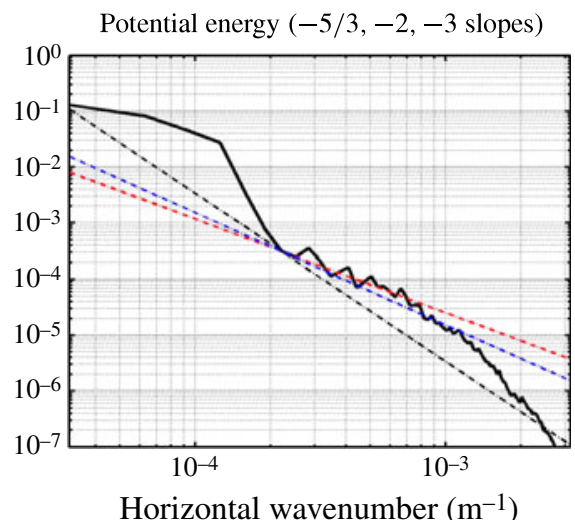

(b)

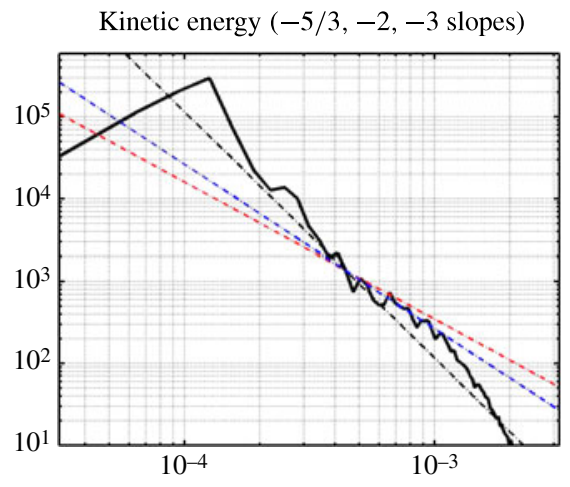

Horizontal wavenumber $\left(\mathrm{m}^{-1}\right)$

FIGURE 15. (Colour online) Run B (variable stratification): spectra averaged around the critical level over a depth of $100 \mathrm{~m}$ of potential energy $(a)$ and kinetic energy $(b)$.

and Gilbert (1988), who showed that spectral laws for tracer-like distributions could be significantly steeper than -1 for flows involving compact (or coherent) structures. The detailed analytical model of Gilbert (1988), building on the earlier work of Lundgren (1982) for the $-5 / 3$ law of three-dimensional turbulence, elaborates that the range of spectral laws is precisely limited to the - [5/3-2] range for the case of a spiral advection of a tracer field by a compact two-dimensional vortex.

One could argue that an alternative explanation for the $-5 / 3$ scaling of vortex stretching is that it could originate from the sharp jump in PV, and also in total $\mathscr{N}^{2}$, caused by the existence of the critical level, as in the case of the surface quasi-geostrophic (SQG) model of Held et al. (1995) pertaining to the strong change in stratification near the tropopause. In such a framework, scaling laws in $-5 / 3$ are predicted for the active tracer (surface temperature) as well as for the potential and kinetic energy (Blumen 1978). However, in contrast to the SQG model, where the jump in $\mathscr{N}^{2}$ corresponds to a jump in the background stratification, here the PV jump is moreover also caused by vortex base state and by the layering formation. The critical level of a compact vortex lens thus corresponds to a more complex setup than for the idealized SQG framework.

\subsubsection{Kinetic and potential energy scaling laws}

Horizontal spectral laws between $-5 / 3$ and -2 for both potential and kinetic energy are also found near the critical levels (see figure $15 a, b$ ), but they concern a significantly narrower range of scales $(7-35 \mathrm{~km})$ than for the vertical derivative of density. Moreover, this shallowing of the energy spectra is significantly more pronounced for a variable background stratification (run B) than for the constant background stratification case (run A, not shown). Such a result points towards a SQG-like influence on potential and kinetic energy of the combined effects of a sharp variation of background stratification (see figure 5) and of the existence of a critical level.

The numerical simulations results for variable stratification agree with the observations of figure $3(a, b)$, where the scaling laws are found for more than a decade of wavenumbers in acoustic reflectivity and they cover less than a decade for the power density spectrum of temperature. 


\subsubsection{Energy and enstrophy fluxes}

From the quasi-geostrophic equations of motion

$$
\begin{aligned}
\frac{\partial \nabla^{2} \Psi}{\partial t} & =-J\left(\Psi, \nabla^{2} \Psi\right)+f \frac{\partial}{\partial z} w, \\
\frac{\partial b}{\partial t} & =-J(\Psi, b)-\mathscr{N}^{2} w,
\end{aligned}
$$

where $b \equiv f(\partial \Psi / \partial z)$ is the buoyancy and $w$ is the vertical velocity, the level-wise kinetic $\left(K E \equiv|\nabla \Psi|^{2} / 2\right)$ and potential energy $\left(P E \equiv b^{2} /\left(2 \mathscr{N}^{2}\right)\right)$ budgets are computed at a given level $z$ as a function of horizontal wavenumber space $k_{h}, k_{h} \equiv\left(k^{2}+l^{2}\right)^{1 / 2}$, where $k, l$ are the zonal and meridional wavenumbers:

$$
\begin{gathered}
\frac{\partial \widehat{K E}}{\partial t}=\operatorname{Re}\left[\widehat{\Psi}^{*} J\left(\widehat{\Psi, \nabla^{2}} \Psi\right)-f \frac{\partial}{\partial z} \widehat{\Psi}^{*} \widehat{w}+\widehat{w} \widehat{b}^{*}\right], \\
\frac{\partial \widehat{P E}}{\partial t}=\operatorname{Re}\left[-\mathscr{N}^{2} \widehat{b}^{*} \widehat{J(\Psi, b)}-\widehat{w}^{*}\right],
\end{gathered}
$$

Here, $\{$ denotes the horizontal spectral transform, the star $*$ denotes the complex conjugate and $\mathrm{Re}$ is the real part. Vertical velocity is obtained through the quasigeostrophic so-called $\omega$-equation (Hoskins, Draghici \& Davies 1978).

The level-wise potential enstrophy $(P \mathcal{Z})$ budget in horizontal wavenumber space is evaluated through

$$
\frac{\partial P \mathcal{Z}}{\partial t}=\operatorname{Re}\left[-\widehat{Q}^{*} \widehat{J(\Psi, Q)}\right]
$$

Level-wise horizontal spectral fluxes $\Pi\left(k_{h}, z\right)$ of potential/kinetic energy and potential enstrophy are evaluated as (see e.g. Frisch 1995) $\Pi\left(k_{h}, z\right)=\int_{k_{h}}^{\infty} T\left(k_{h}, z\right) \mathrm{d} k_{h}$, where $T\left(k_{h}, z\right)$ corresponds to the right-hand side terms in (3.10)-(3.12).

Energy and enstrophy fluxes are plotted (in variance preserving form) for run A in figure 16 for 10 revolution periods of the initial eddy. Note, however, that statistics have not converged for the largest spatial scales in the 70-200 km range because we are dealing with a spindown simulation and, thus, the time span of the statistics is necessarily limited when compared with a forced/damped simulation in statistical steady state. For the entire domain statistics, negative energy fluxes in the 20-70 km range reveal that the mesoscale range is being depleted by baroclinic instability to the benefit of the submesoscale range $(2-10 \mathrm{~km}$; see figure $16 a)$. Figure $16(c)$ documents the level-wise statistics of energy fluxes. Positive sign fluxes at submesoscales tend to concentrate around the critical levels, aloft $(800 \mathrm{~m})$ and beneath the eddy core $(1600 \mathrm{~m})$, and are also strong at mid-depth of the eddy. This diagnoses a direct energy flux from the mesoscale range to the submesoscales, corresponding to the formation of layering at the critical levels (figure 8) and to the tiny eddies seen at mid-depth (figure 7) resulting from the destabilization of the long filaments, respectively.

Enstrophy fluxes (see figure 16b) indicate that the $5-50 \mathrm{~km}$ range of scales undergoes a nearly constant enstrophy flux to small scales, characteristic of a direct enstrophy cascade. Level-wise, details of the enstrophy fluxes (see figure 16d) keep a signature of layering patterns in enstrophy fluxes around 800 and $1600 \mathrm{~m}$.

Note here that despite positive fluxes toward small scales, we have diagnosed essentially no energy dissipation ( $0.5 \%$ of the total energy after 250 days) but there is a substantial enstrophy dissipation ( $20 \%$ is dissipated after 250 days) as expected in a quasi-geostrophic dynamics. 
(a)

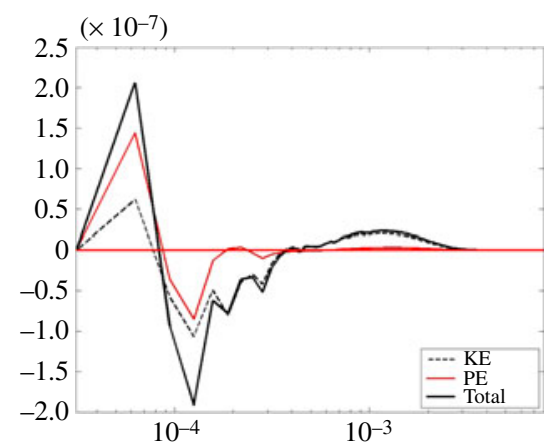

(c)

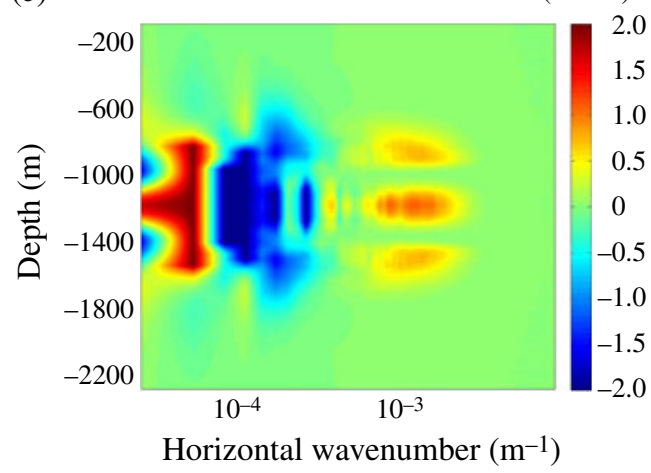

(b)

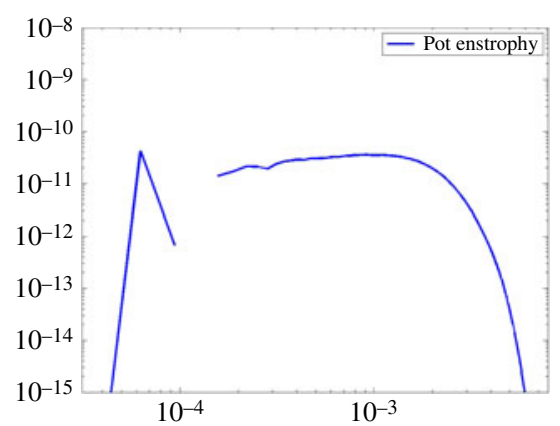

$(d)$

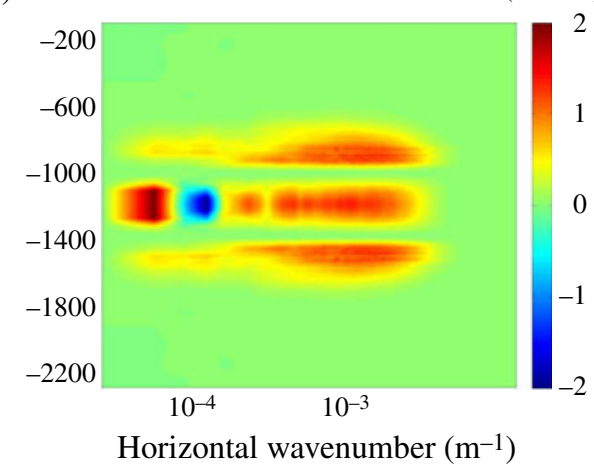

FIGURE 16. Run A. Energy fluxes: entire domain statistics $\int \pi\left(k_{h}, z\right) \mathrm{d} z(a)$ and level-wise $\Pi\left(k_{h}, z\right)(c)$. Both $(a)$ and $(c)$ are plotted in variance-preserving form, such that fluxes are multiplied by the horizontal wavenumber. Enstrophy fluxes: entire domain statistics $\int \pi\left(k_{h}, z\right) \mathrm{d} z(b)$ and level-wise $\Pi\left(k_{h}, z\right)(d)$. In all plots the largest horizontal scale is $200 \mathrm{~km}$.

Similar results are obtained for the depth-variable stratification (run B, figure 17), but with a significant widening of the range of both submesoscale direct energy flux $(1.5-15 \mathrm{~km})$ and of direct enstrophy cascade $(4-100 \mathrm{~km})$. Moreover, there is a strong amplification of both energy and enstrophy fluxes at the main thermocline level, where the layering presents marked fine vertical scales (see figure 9).

In the idealized SQG set-up, the $-5 / 3$ scaling law corresponds to an inertial range with a constant potential energy flux $\Pi\left(k_{h}\right)$ to small horizontal scales (see the discussion by Capet et al. (2008) on the differences between SQG $K E$ and $P E$ advective fluxes). Here, the ranges of wavenumbers displaying a near $-5 / 3$ scaling in potential/kinetic energy for run B do not coincide with a positive flux $\Pi\left(k_{h}\right)$ in either kinetic or potential energy, but correspond instead to a positive production term $T\left(k_{h}\right)$, as corroborated by the positive slope of the flux curves for the $7-35 \mathrm{~km}$ range in figure $17(a, c)$. Our results for $K E$ and $P E$ fluxes do not strictly conform to the predictions of the idealized SQG framework.

\section{Discussion}

Inside the layering aloft and below a Meddy, in situ data display a $\left[\begin{array}{ll}-5 / 3 & -2\end{array}\right]$ scaling law of horizontal scales for two different quantities, temperature and its vertical derivative (obtained from the deconvoluted seismic signal). These scalings 
(a)

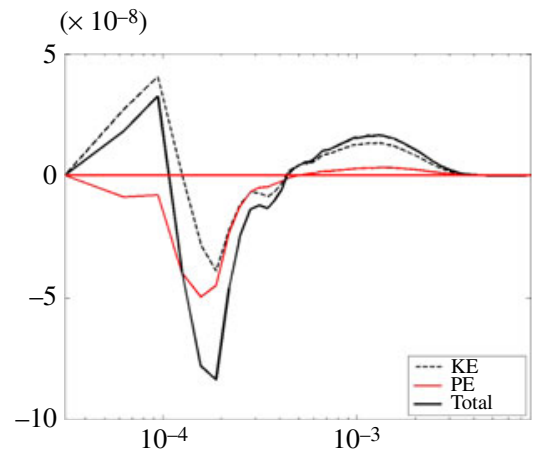

$(c)$

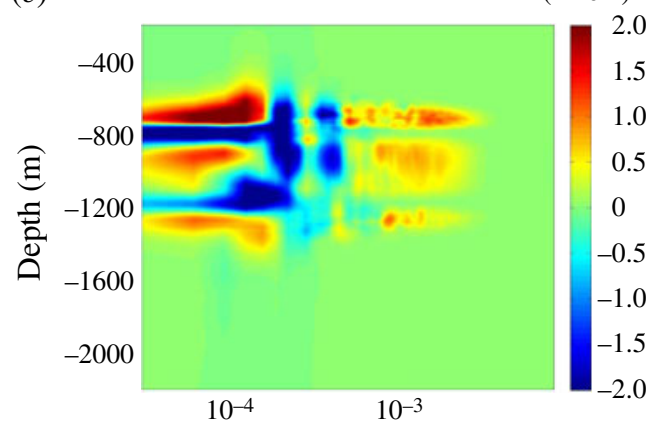

Horizontal wavenumber $\left(\mathrm{m}^{-1}\right)$ (b)

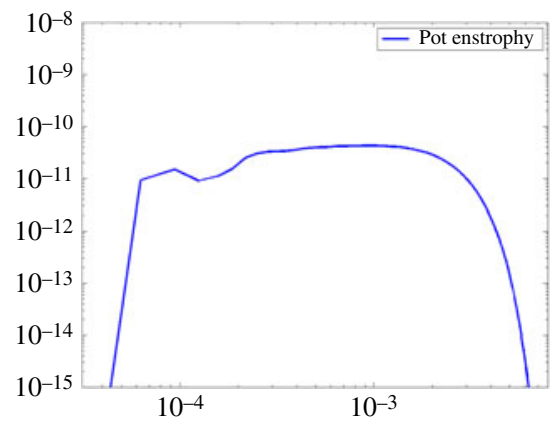

$(d)$

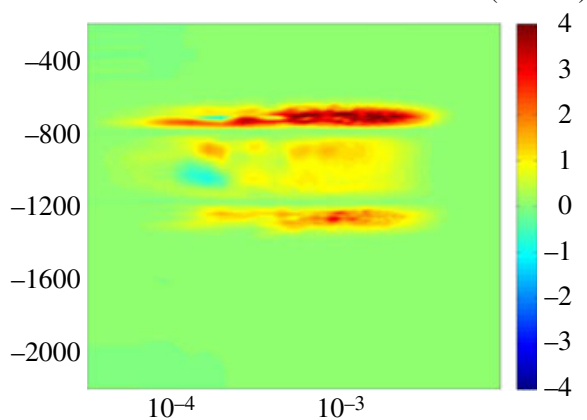

Horizontal wavenumber $\left(\mathrm{m}^{-1}\right)$

FIGURE 17. Run B. Energy fluxes: entire domain statistics $\int \pi\left(k_{h}, z\right) \mathrm{d} z(a)$ and level-wise $\Pi\left(k_{h}, z\right)(c)$. Both $(a)$ and $(c)$ are plotted in variance-preserving form, such that fluxes are multiplied by the horizontal wavenumber. Enstrophy fluxes: entire domain statistics $\int \pi\left(k_{h}, z\right) \mathrm{d} z(b)$ and level-wise $\Pi\left(k_{h}, z\right)(d)$. In all plots the horizontal scale is $200 \mathrm{~km}$.

correspond to two different ranges of wavelengths, respectively between 5 and $50 \mathrm{~km}$ for temperature, and between $500 \mathrm{~m}$ and $5 \mathrm{~km}$ for its vertical derivative.

The scaling law for the observed temperature spectrum can be related to the Garret and Munk spectrum (Samelson \& Paulson 1988). However, in this paper, we explore the dynamics underlying the layering formation mechanism, through the slow dynamics captured by quasi-geostrophic equations. Three-dimensional high resolution numerical simulations of the destabilization of a lens-shaped vortex confirm that the vertical stacking of sharp jumps in density at its periphery is the three-dimensional analogue of the preferential wind-up of PV near a critical radius, a phenomenon which has been documented for barotropic vortices. For a small-Burger (flat) lens vortex, baroclinic instability ensures a sustained growth rate of sharp jumps in temperature near the critical levels of the leading unstable modes. Such results can be obtained for a background stratification which is due to temperature only and does not require the existence of salt anomalies.

Aloft and beneath the vortex core, numerical simulations robustly reproduce the $k_{h}^{-5 / 3}$ scaling law of horizontal scales for the vertical gradient of temperature that is observed in situ inside the layering, whatever the background Brunt-Väisälä profile. Such a result stems from the tracer-like behaviour of the vortex stretching component, which dominates over the relative vorticity component in the quasi-geostrophic PV 
and which is proportional to the vertical gradient of temperature. Previous studies, e.g. Gilbert (1988), have shown that spectra of tracer fields can be steeper than -1 , namely in $-5 / 3$ or -2 , if the advection field is very compact spatially, with a $-5 / 3$ slope corresponding to a spiral advection of tracer. Such a scaling law could thus stem from a geometric origin.

The simulated scaling laws for kinetic and potential energy also reveal a narrower range of $-5 / 3$ to -2 scaling near the critical levels, which is strongly enhanced for a sharply variable background Brunt-Väisälä profile, such as observed in situ. This raises the possibility of another plausible mechanism leading to $-5 / 3$ scalings for the power spectra of tracer field as well as for horizontal velocity and buoyancy, namely SQG-like dynamics à la Held et al. (1995). The SQG framework posits a sharp jump in background stratification, while in the present case, we have investigated the cases of both a constant and variable background stratification, onto which variations of effective $\mathscr{N}^{2}$ can be caused by the compact vortex lens base state and/or can also be attributed to the layering that builds up near the critical level.

A detailed examination of the energy fluxes reveal that the near $-5 / 3$ scaling in $K E$ and $P E$ do not correspond to a direct flux to smaller scales but to a positive production of energy at smaller scales than the vortex lens scale.

Our study differs from previous investigations of geophysical flows with $-5 / 3$ scaling (Molemaker et al. 2010; Vallgren et al. 2011) in that we are dealing with a deterministic single vortex of moderate amplitude, moreover using quasi-geostrophic dynamics. Our submesoscale production does not therefore require ageostrophic dynamics and the small lateral scales can be interpreted as corresponding to local small radii of deformation: the high vertical resolution in our numerical simulations is essential to quantitatively reach such a regime, since horizontal and vertical scales are tied by Charney's (1971) isotropy.

Energy and enstrophy fluxes emphasize a strong production of submesoscales and a kinetic and potential energy flux from mesoscale to submesoscales range near the critical levels. Such horizontal submesoscale production, which is correlated to the accumulation of thin vertical scales inside the layering, thus has a significant slow dynamical component, well-captured by quasi-geostrophy.

Nevertheless, caution has to be taken while comparing the density gradient with temperature gradients issued from seismic data. Here, we did not take into account the compensation effect of salt on the observed fine-scale structures. Results of this first study will be compared in a following study to those of numerical simulations enabling: (i) salt compensating effects in the equation of state; and (ii) fast dynamics, using Boussinesq equations.

\section{Acknowledgements}

This work has been made possible through grant 96130 of IDRIS (France), through the memorandum of understanding signed between IFREMER and JAMSTEC, enabling access to Earth Simulator (Yokohama, Japan) and through the support of grants 'Ocean LAyering' and 'SIMILA' of Agence Nationale de la Recherche (SIMI 5-6, 2011, PDOC 2011). We thank reviewers for their insightful comments. This paper is dedicated to the memory of professor B.L.H.

\section{Appendix. Numerics}

Boundary conditions used in the present simulations correspond to rigid lids at top and bottom of the domain and to horizontal doubly periodic conditions. 
The pseudospectral code of Hua \& Haidvogel (1986), was originally entirely formulated in horizontal Fourier space and in vertical modes, both for the linear and nonlinear advection terms. The main change is the current version is that nonlinear terms are now calculated at $z$-levels, rather than through a triple interaction between vertical modes (e.g. Hua \& Haidvogel 1986, (2.4)). Time stepping is still performed in Fourier/vertical modes, but the streamfunction field is now projected back to physical vertical space before evaluating the nonlinear advection terms. This modification of the code lowers the computational cost from a $N_{v}^{3}$ to a $N_{v}^{2}$ law, where $N_{v}$ is the number of levels/vertical modes used in the simulation, for the case of a depth-variable stratification $\mathscr{N}(z)$.

Specifically, the streamfunction at a given level $z$ is expanded in horizontal Fourier series

$$
\Psi(x, y, z, t)=\sum_{k=-N_{h} / 2}^{k=N_{h} / 2} \sum_{l=-N_{h} / 2}^{l=N_{h} / 2} \widehat{\Psi}\left(\boldsymbol{k}_{\boldsymbol{h}}, z, t\right) \exp [\mathrm{i}(k x+l y)],
$$

where $\boldsymbol{k}_{\boldsymbol{h}} \equiv(k, l)$ and $N_{h}$ is the resolution in a given horizontal direction.

For a depth-variable stratification $\mathscr{N}(z)$, vertical modes are computed from

$$
\frac{\partial}{\partial z}\left(\frac{f^{2}}{\mathscr{N}^{2}(z)} \frac{\partial}{\partial z} F_{m}(z)\right)=-\lambda_{m}^{2} F_{m}(z)
$$

with top and bottom boundary conditions $(\partial / \partial z) F_{m}(z)=0$ at $z=0,-H$. Projecting the streamfunction onto the vertical mode $m$ yields

$$
\widehat{\Psi}\left(\boldsymbol{k}_{\boldsymbol{h}}, m, t\right)=\int_{-H}^{0} \widehat{\Psi}\left(\boldsymbol{k}_{\boldsymbol{h}}, z, t\right) F_{m}(z) \mathrm{d} z,
$$

while $\mathrm{PV}$ in mode $m$ is given by

$$
\begin{aligned}
\widehat{Q}\left(\boldsymbol{k}_{\boldsymbol{h}}, m, t\right) & =\int_{-H}^{0} \widehat{Q}\left(\boldsymbol{k}_{\boldsymbol{h}}, z, t\right) F_{m}(z) \mathrm{d} z, \\
& \left.=-\left(k^{2}+l^{2}+\lambda_{m}^{2}\right) \Psi \widehat{\left(\boldsymbol{k}_{\boldsymbol{h}}, m\right.}, t\right) .
\end{aligned}
$$

Time stepping is performed in horizontal Fourier space and in vertical modes, through PV conservation in (3.1):

$$
\frac{\partial}{\partial t} \widehat{Q}\left(\boldsymbol{k}_{\boldsymbol{h}}, m, t\right)+[\widehat{\nabla \cdot \boldsymbol{u} Q}]\left(\boldsymbol{k}_{\boldsymbol{h}}, m, t\right)=-v k_{h}^{6} \widehat{Q}\left(\boldsymbol{k}_{\boldsymbol{h}}, m, t\right)
$$

with $\boldsymbol{u}=\boldsymbol{e}_{z} \times \nabla \Psi$. Hyperviscosity effects are calculated by exact integration via the use of an integrating factor technique (Canuto et al. 1988):

$$
\widehat{Q}\left(\boldsymbol{k}_{\boldsymbol{h}}, m, t+\Delta t\right)=\mathrm{e}^{-v k_{h}^{6} \Delta t} \widehat{Q}\left(\boldsymbol{k}_{\boldsymbol{h}}, m, t\right)+\Delta \widehat{t}\left(\boldsymbol{k}_{\boldsymbol{h}}, m, t\right) .
$$

At each time step, the modal streamfunction field is projected back to $z$ space and the vortex stretching component of PV is evaluated through finite differences in $z$. For nonlinear terms, products are first calculated in real horizontal/vertical space $(x, y, z)$, before being transformed to horizontal Fourier space

$$
\left.\widehat{J}\left(\boldsymbol{k}_{h}, z, t\right)=-\widehat{\nabla \cdot(\boldsymbol{u} Q}\right),
$$


and are then projected onto the vertical mode $m$

$$
\widehat{J}\left(\boldsymbol{k}_{\boldsymbol{h}}, m, t\right)=\int_{-H}^{0} \widehat{J}\left(\boldsymbol{k}_{\boldsymbol{h}}, z, t\right) F_{m}(z) \mathrm{d} z .
$$

The code is parallelized in MPI along the $z$ direction and has been customized to the architecture of Earth Simulator 2 which is a massively parallel vector computer. We take advantage of the vector performances of individual processors for fast Fourier transforms in the horizontal direction. Parallelization in the $z$ coordinate is welladapted to our simulations which emphasize high resolution in the vertical direction.

\section{REFERENCES}

Armi, L., Hebert, D., OAkey, N., Price, J. F., Richardson, P. I., Rossby, H. T. \& RUDDICK, B. 1989 Two years in a life of a Mediterranean salt lens. J. Phys. Oceanogr. 19, 354-370.

Balmforth, N., Smith, S. G. L. \& Young, W. R. 1998 Dynamics of interfaces and layers in a stratified turbulent fluid. J. Fluid Mech. 355, 329-358.

Balmforth, N., Smith, S. G. L. \& Young, W. R. 2001 Disturbing vortices. J. Fluid Mech. 426, 95-133.

Biescas, B., Sallars, V., Pelegri, J. L., Machin, F., Carbonell, R., Buffett, G., DANOBEITIA, J. J. \& CALAhORRANO, A. 2008 Imaging meddy finestructure using multichannel seismic reflection data. Geophys. Res. Lett. 35, L033971.

Blumen, W. 1978 Uniform potential vorticity flow. Part I. Theory of wave interactions and two-dimensional turbulence. J. Atmos. Sci. 35, 774-783.

Brethouwer, G., Billant, P., Lindborg, E. \& Chomaz, J.-M. 2007 Scaling analysis and simulation of strongly stratified turbulent flows. J. Fluid Mech. 585, 343-368.

Canuto, C., Hussaini, M. Y., Quarteroni, A. \& Zane, T. A. 1988 Spectral Methods in Fluid Dynamics. Springer.

Capet, X., Klein, P., Hua, B. L., Lapeyre, G. \& McWilliams, J. C. 2008 Surface kinetic energy transfer in surface quasi-geostrophic flows. J. Fluid Mech. 604 (1), 165-174.

Charney, J. 1971 Geostrophic turbulence. J. Atmos. Sci. 28, 1087-1095.

Dritschel, D. G. \& DE LA TORRe Juarez, M. 1996 The instability and breakdown of tall columnar vortices in a quasi-geostrophic fluid. J. Fluid Mech. 328, 129-160.

FrISCH, U. 1995 Turbulence. Cambridge University Press.

Geli, L., Cosquer, E., Hobbs, R. W., Klaeschen, D., Papenberg, C., Thomas, Y., MENesguen, C. \& HuA, B. L. 2009 High resolution seismic imaging of the ocean structure using a small volume airgun source array in the Gulf of Cadiz. Geophys. Res. Lett. 36, L00D09.

Gent, P. \& MCWilliams, J. C. 1986 The instability of barotropic circular vortices. Geophys. Astrophys. Fluid Dyn. 35, 209-233.

Gilbert, A. D. 1988 Spiral structures and spectra in two-dimensional turbulence. J. Fluid Mech. 193, 475-497.

Golub, G. H. \& Van LoAn, C. F. 1996 Matrix Computations, vol. 10. Johns Hopkins Studies in the Mathematical Sciences, The Johns Hopkins University Press.

Gonella, J. \& Michon, D. 1988 Ondes internes profondes révélées par sismique réflexion au sein des masses d'eau en Atlantique-Est. C. R. Acad. Sci. Ser. II 306 (12), 781-787.

Hamilton, K., Takahashi, Y. O. \& Ohfuchi, W. 2008 Mesoscale spectrum of atmospheric motions investigated in a very fine resolution global general circulation model. J. Geophys. Res. 113, D18110.

Held, I. M., Pierrehumbert, R. T., Garner, S. T. \& Swanson, K. L. 1995 Surface quasi-geostrophic dynamics. J. Fluid Mech. 282, 1-20.

HоввS, R. 2007 GO (geophysical oceanography): a new tool to understand the thermal structure and dynamics of oceans. European Union Newsletter 2, http://www.aapg.org/europe/newsletters/ 2007/06jun/06jun07europe.pdf. 
Holbrook, W. S. \& Fer, I. 2005 Ocean internal wave spectra inferred from seismic reflection transects. Geophys. Res. Lett. 32, L15604.

Holbrook, W. S., Páramo, P., Pearse, S. \& Schmitt, R. W. 2003 Thermohaline fine structure in an oceanographic front from seismic reflection profiling. Science 301, 821-824.

Hoskins, B. J., Draghici, I. \& Davies, H. C. 1978 A new look at the $\omega$-equation. $Q$. J. $R$. Meteorol. Soc. 104, 31-38.

Hua, B. L. \& Haidvogel, D. B. 1986 Numerical simulations of the vertical structure of quasi-geostrophic turbulence. J. Atmos. Sci. 43, 2923-2936.

Klein, P., Hua, B., Lapeyre, G., Capet, X., Gentil, S. L. \& Sasaki, H. 2008 Upper ocean turbulence from high-resolution 3D simulations. J. Phys. Oceanogr. 38, 1748-1763.

Lapeyre, G. \& Klein, P. 2006 Dynamics of the upper oceanic layers in terms of surface quasigeostrophy theory. J. Phys. Oceanogr. 36, 165-176.

LindBorg, E. 2005 The effect of rotation on the mesoscale energy cascade in the free atmosphere. Geophys. Res. Lett. 32, L01809.

Lundgren, T. 1982 Strained spiral vortex model for turbulent fine structure. Phys. Fluids 25 (12), 2193-2203.

McIntyre, M. 1970 Diffusive destabilisation of the baroclinic circular vortex. Geophys. Fluid Dyn. 1, 19-57.

Ménesguen, C., Hua, B., Carton, X., Klingelhoefer, F., Schnürle, P. \& Reichert, C. 2012 Arms winding around a meddy seen in seismic reflection data close to the Morocco coastline. Geophys. Res. Lett. 39, L05604.

Ménesguen, C., Hua, B., Papenberg, C., Klaeschen, D., Geli, L. \& Hobbs, R. 2009 Effect of bandwidth on seismic imaging of rotating stratified turbulence surrounding an anticyclonic eddy from field data and numerical simulations. Geophys. Res. Lett. 36, L00D05.

Molemaker, M. J., MCWilliams, J. C. \& CAPET, X. 2010 Balanced and unbalanced routes to dissipation in an equilibrated eady flow. J. Fluid Mech. 654, 35-63.

Nandi, P., Holbrook, W., Pearse, S., Paramo, P. \& Schmitt, R. 2004 Seismic reflection imaging of water mass boundary in the Norwegian Sea. Geophys. Res. Lett. 31, L23311.

NAströM, G. D. \& GAGE, K. S. 1985 A climatology of atmospheric wavenumber spectra of wind and temperature observed by commercial aircraft. J. Atmos. Sci. 42, 950-960.

Nguyen, H. Y., HuA, B. L., Schopp, R. \& CARton, X. 2012 Slow quasigeostrophic unstable modes of a lens vortex in a continuously stratified flow. Geophys. Astrophys. Fluid Dyn. 106 (3), 305-319.

Papenberg, C., Klaeschen, D., Krahmann, G. \& Hobbs, R. W. 2010 Ocean temperature and salinity inverted from combined hydrographic and seismic data. Geophys. Res. Lett. 37, L04601 doi:10.1029/2009GL042115.

Pedlosky, J. 1996 Ocean Circulation Theory. Springer.

PingReE, R. D. \& LE CANn, B. 1993 A shallow MEDDY (a SMEDDY) from the secondary mediterranean salinity maximum. J. Geophys. Res. 982, 20169-20186.

Pinheiro, L. M., Song, H., Ruddick, B., Dubert, J., Ambar, I., Mustafa, K. \& Bezerra, R. 2010 Detailed 2-D imaging of the mediterranean outflow and Meddies off W Iberia from multichannel seismic data. J. Mar. Syst. 79 (12), 89-100.

Richardson, P., Bower, A. \& Zenk, W. 2000 A census of Meddies tracked by floats. Prog. Oceanogr. 45, 209-250.

RILEY, J. \& LINDBORG, E. 2008 Stratified turbulence: a possible interpretation of some geophysical turbulence measurements. J. Atmos. Sci. 65, 2416-2424.

Ruddick, B. R. \& Richards, K. J. 2003 Oceanic thermohaline intrusions: observations. Prog. Oceanogr. 56 (3-4), 499-527.

SAFFMAN, P. 1971 On the spectrum and decay of random two-dimensional vorticity distributions at large Reynolds number. Stud. Appl. Maths 50, 377-383.

Sallarès, V., Biescas, B., Buffett, G., Carbonell, R., Dañobeitia, J. J. \& Pelegrí, J. L. 2009 Relative contribution of temperature and salinity to ocean acoustic reflectivity. Geophys. Res. Lett. 36, L00D06.

SAMElson, R. M. \& PAUlson, C. A. 1988 Towed thermistor chain observations of fronts in the subtropical North Pacific. J. Geophys. Res.: Oceans 93 (C3), 2237-2246. 
Smith, K. S. \& Ferrari, R. 2009 The production and dissipation of compensated thermohaline variance by mesoscale stirring. J. Phys. Oceanogr. 39, 2477-2501.

Vallgren, A., Deusebio, E. \& Lindborg, E. 2011 Possible explanation of the atmospheric kinetic and potential energy spectra. Phys. Rev. Lett. 107, 268501.

Waite, M. \& BARTello, P. 2004 Stratified turbulence dominated by vortical motion. J. Fluid Mech. 517, 281-308. 